\title{
On a permutation group related to $\zeta(2)$
}

\author{
by \\ Georges Rhin (Metz) and Carlo Viola (Pisa)
}

1. Introduction. Ever since the appearance of Beukers' paper [1], it was clear that double integrals of the type

$$
\int_{0}^{1} \int_{0}^{1} P(x, y) \frac{d x d y}{1-x y}
$$

where $P(x, y) \in \mathbb{Z}[x, y]$, are relevant to the study of Diophantine properties of $\zeta(2)=\sum_{n=1}^{\infty} n^{-2}=\pi^{2} / 6$, although the problem of finding polynomials $P(x, y)$ yielding good irrationality measures of $\zeta(2)$ proved to be a difficult one. With the choice

$$
P(x, y)=L_{n}(x)(1-y)^{n},
$$

where

$$
L_{n}(x)=\frac{1}{n !} \frac{d^{n}}{d x^{n}}\left(x^{n}(1-x)^{n}\right)
$$

is the $n$th Legendre polynomial, Beukers obtained the sequence of rational approximations to $\zeta(2)$ previously found by Apéry through an entirely different method, and hence gave a new proof of Apéry's result that

$$
\frac{10 \log \frac{\sqrt{5}+1}{2}}{5 \log \frac{\sqrt{5}+1}{2}-2}=11.85078 \ldots
$$

is an irrationality measure of $\zeta(2)$.

We recall that $\lambda$ is an irrationality measure of the irrational number $\alpha$ if for any $\varepsilon>0$ there exists a constant $q_{0}=q_{0}(\varepsilon)>0$ such that

$$
\left|\alpha-\frac{p}{q}\right|>q^{-\lambda-\varepsilon}
$$

for all integers $p$ and $q$ with $q>q_{0}$. We denote by $\mu(\alpha)$ the minimum of such exponents $\lambda$. 
By repeated partial integration, one sees that

$$
\begin{aligned}
& \int_{0}^{1} \int_{0}^{1} L_{n}(x)(1-y)^{n} \frac{d x d y}{1-x y} \\
&=(-1)^{n} \int_{0}^{1} \int_{0}^{1}\left(\frac{x(1-x) y(1-y)}{1-x y}\right)^{n} \frac{d x d y}{1-x y},
\end{aligned}
$$

and the left side of (1.2) is easily seen to be $a_{n}-b_{n} \zeta(2)$, with $b_{n}$ integer and $a_{n}$ a rational number having a controlled denominator, while the right side of (1.2) is suitable to get asymptotic estimates of $a_{n}-b_{n} \zeta(2)$ and $b_{n}$ as $n \rightarrow \infty$.

The search for better polynomials $P(x, y)$ in (1.1) was not pursued until the appearance of the paper [3] by Dvornicich and Viola, where the irrationality measure $\mu(\zeta(2))<10.0298$ was obtained. This was later improved to $\mu(\zeta(2))<7.5252$ by Hata [5] and to $\mu(\zeta(2))<7.398537$ by Rhin and Viola [8]. In a recent paper, Hata [7] considered the integral

$$
\int_{0}^{1} \int_{0}^{1}\left(\frac{x^{15}(1-x)^{15} y^{14}(1-y)^{14}}{(1-x y)^{12}}\right)^{n} \frac{d x d y}{1-x y},
$$

and transformed it into an integral of the type (1.1) by $12 n$-fold partial integration, thus proving that (1.3) has indeed the form $a_{n}-b_{n} \zeta(2)$ with $a_{n} \in \mathbb{Q}$ and $b_{n} \in \mathbb{Z}$. By making use of the $p$-adic valuation of suitable binomial coefficients, Hata showed that the denominator of $a_{n}$ is relatively small, and proved the remarkable result $\mu(\zeta(2))<6.3489$. He subsequently improved this to $\mu(\zeta(2))<5.687$ ([7], Addendum) by combining his method with the properties of a birational transformation of the plane introduced in $[8]$.

The purpose of the present paper is the arithmetical study of a family of integrals generalizing (1.3). We define

$$
I(h, i, j, k, l)=\int_{0}^{1} \int_{0}^{1} \frac{x^{h}(1-x)^{i} y^{k}(1-y)^{j}}{(1-x y)^{i+j-l}} \frac{d x d y}{1-x y},
$$

where $h, i, j, k, l$ are any non-negative integers, and we show that the Diophantine properties of the integrals (1.4) rest upon an underlying algebraic structure. Specifically, we consider the action on the integrals (1.4) of a naturally arising permutation group.

Some algebraic properties of the integrals (1.4) (in the more general case where $h, i, j, k, l$ are any complex parameters with real parts $>-1$ ) were studied in 1905 by A. C. Dixon [2] who, however, gave no arithmetical applications of them. Dixon found that the value of (1.4) is unchanged under a cyclic permutation of $h, i, j, k, l$. This can be shown e.g. by applying to the 
integral (1.4) the birational transformation

$$
\tau:\left\{\begin{array}{l}
\xi=\frac{1-x}{1-x y}, \\
\eta=1-x y,
\end{array}\right.
$$

defined in [8]. Moreover, Dixon remarked that the Euler integral representation of Gauss's hypergeometric function shows that the quantity

$$
\frac{I(h, i, j, k, l)}{\Gamma(h+1) \Gamma(i+1) \Gamma(j+1) \Gamma(k+1) \Gamma(l+1)}
$$

is a symmetric function of the sums $h+i, i+j, j+k, k+l, l+h$. In our context the gamma-factors are factorials, and they provide information on the $p$-adic valuation of the rational part $a_{n}$ of

$$
\begin{aligned}
I(h n, i n, j n, k n, l n) & =\int_{0}^{1} \int_{0}^{1}\left(\frac{x^{h}(1-x)^{i} y^{k}(1-y)^{j}}{(1-x y)^{i+j-l}}\right)^{n} \frac{d x d y}{1-x y} \\
& =a_{n}-b_{n} \zeta(2) .
\end{aligned}
$$

A crucial step to obtain this is to characterize in terms of quotients of factorials the (left) cosets of a subgroup $\mathbf{T}$, related to the transformation (1.5), in a permutation group $\boldsymbol{\Phi}$ related to the hypergeometric integral transformation $\varphi$ which leaves the value of (1.6) unchanged, and we do this in Section 3.

We remark that if $i+j-l>\min \{h, i, j, k\},(1.4)$ cannot be transformed by partial integration into an integral of the type (1.1) to which Hata's arithmetical method [7] can be successfully applied, and in fact if $i+j-l>$ $\min \{h, k\}$, by the partial integration method it is not even clear that (1.4) equals $a-b \zeta(2)$ for some $a \in \mathbb{Q}$ and $b \in \mathbb{Z}$. Therefore, in order to apply for any admissible choice of $h, i, j, k, l$ the whole group $\boldsymbol{\Phi}$ to the study of the $p$-adic valuation of the rational part $a_{n}$ of (1.7), we need first to get rid of the partial integration method, and to show that (1.4) has the form $a-b \zeta(2)$ without using a representation of the type (1.1). In Section 2 we achieve this, and we find an (optimal) pair of non-negative integers $M, N$ such that $d_{M} d_{N} a \in \mathbb{Z}$ (we denote $d_{m}=$ l.c.m. $\{1,2, \ldots, m\}$ ).

The choice $h=i=12, j=k=14, l=13$ in (1.7) allows us to prove the irrationality measure

$$
\mu(\zeta(2))<5.441243 \text {. }
$$

We incidentally remark that the one-dimensional analogue of our method applies to the integrals

$$
\int_{0}^{1}\left(\frac{x^{h}(1-x)^{i}}{(1+x)^{i-l}}\right)^{n} \frac{d x}{1+x},
$$

where $h, i, l$ are any integers satisfying $h>\max \{0,-l\}, i>\max \{0, l\}$, and allows one to obtain irrationality measures of $\log 2$. Here the choice 
$h=i=7, l=1$ yields the same sequence of rational approximations to $\log 2$ found by Rukhadze [9] (see also Hata [5]), and hence the bound

$$
\mu(\log 2)<3.89139978 .
$$

This paper is organized as follows. In Section 2 we simplify and generalize a method introduced in our previous paper ([8], Section 3). Thus we prove some arithmetical lemmas showing that for any non-negative integers $h$, $i, j, k, l$ we have

$$
I(h, i, j, k, l)=a-b \zeta(2)
$$

with $b \in \mathbb{Z}$ and $d_{M} d_{N} a \in \mathbb{Z}$, where $M$ and $N$ are invariant under the action of the transformation (1.5) on $I(h, i, j, k, l)$, and allowing one to express the integer $b$ as a double contour integral.

In Section 3 we apply to $I(h, i, j, k, l)$ the hypergeometric transformation $\varphi$, and we can do this by imposing the restriction that not only

$$
h, i, j, k, l
$$

but also

$$
j+k-h, k+l-i, l+h-j, h+i-k, i+j-l
$$

are non-negative. Denoting by $\sigma$ the transformation that interchanges the variables $x, y$ in the integral $I(h, i, j, k, l)$, we consider the permutations $\varphi$, $\boldsymbol{\tau}$ and $\boldsymbol{\sigma}$ of the ten integers (1.8) and (1.9) induced by the integral transformations $\varphi, \tau$ and $\sigma$ respectively, and we characterize the left cosets of the subgroup $\mathbf{T}=\langle\boldsymbol{\tau}, \boldsymbol{\sigma}\rangle$ in the group $\boldsymbol{\Phi}=\langle\boldsymbol{\varphi}, \boldsymbol{\tau}, \boldsymbol{\sigma}\rangle$ in terms of quotients of factorials.

In Section 4 we analyse the $p$-adic valuation and hence the denominator of $a_{n}$ by a discussion close to Hata's method [7]. Our approach differs from Hata's in the use of the $p$-adic valuation of the factorials occurring in the transformation formulae corresponding to the left cosets of $\mathbf{T}$ in $\mathbf{\Phi}$, in place of Hata's analysis of the $p$-adic valuation of binomial coefficients.

In Section 5 we give the asymptotic estimates of

$$
I(h n, i n, j n, k n, l n)=a_{n}-b_{n} \zeta(2)
$$

and of $b_{n}$ as $n \rightarrow \infty$, under the further restriction that the integers (1.8) and (1.9) are strictly positive, and we prove the irrationality measure $\mu(\zeta(2))<$ 5.441243 mentioned above.

We point out here that, even though the values of the integers (1.8) in Section 5 must be such that (1.8) and (1.9) are strictly positive, we cannot impose this restriction from the very beginning, since our arithmetical lemmas in Section 2 consist in reducing $I(h, i, j, k, l)$ to simpler integrals for which some of the integers (1.8) vanish, or some of (1.9) may be negative. Hence it is essential to restrict successively the signs of (1.8) and (1.9) as indicated above. 
We wish to make a concluding remark on the motivation of this paper. Unlike Diophantine approximation to algebraic numbers, where fairly general methods are available even in the search for effective irrationality measures, the Diophantine theory for transcendental constants is characterized by lack of generality in the tools employed. However, the algebraic approach we work out in this paper also applies to the Diophantine study of a class of constants other than $\zeta(2)$ or $\log 2$, which can be related to suitable integrals. We hope that our paper will be primarily viewed as a contribution to the quest for general methods in the theory of Diophantine approximation to transcendental (or conjecturally transcendental) constants.

We are indebted to F. Amoroso, R. Dvornicich and D. Masser for interesting discussions on some aspects of our results.

2. Arithmetical lemmas. Let $h, i, j, k, l$ be integers. We consider the integral

$$
I(h, i, j, k, l)=\int_{0}^{1} \int_{0}^{1} \frac{x^{h}(1-x)^{i} y^{k}(1-y)^{j}}{(1-x y)^{i+j-l}} \frac{d x d y}{1-x y},
$$

and note that the condition for $I(h, i, j, k, l)$ to be finite is that $h, i, j, k, l$ are all non-negative, which we shall henceforth assume. We recall from [8] that the transformation $\tau$ defined by

$$
\tau:\left\{\begin{array}{l}
\xi=\frac{1-x}{1-x y} \\
\eta=1-x y
\end{array}\right.
$$

has period 5 and maps the unit square $(0,1) \times(0,1)$ onto itself. Moreover, the function

$$
\frac{x(1-x) y(1-y)}{1-x y}
$$

and the measure

$$
\frac{d x d y}{1-x y}
$$

are invariant under the action of $\tau$.

Let $J_{0}=I(h, i, j, k, l)$, and let $J_{1}$ be the integral obtained by applying to $J_{0}$ the transformation $\tau$, i.e. by making in (2.1) the change of variables

$$
\tau^{-1}:\left\{\begin{array}{l}
x=1-\xi \eta \\
y=\frac{1-\eta}{1-\xi \eta}
\end{array}\right.
$$

and then replacing $\xi, \eta$ with $x, y$ respectively. We find $J_{1}=I(i, j, k, l, h)$, so that with the action of $\tau$ on $J_{0}$ we associate the cyclic permutation $\tau$ of $h$, $i, j, k, l$ represented by the diagram 


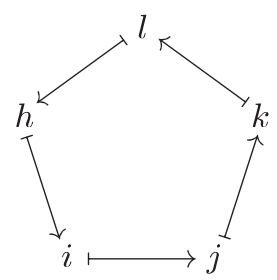

As usual, we denote this permutation by

$$
\boldsymbol{\tau}=\left(\begin{array}{lllll}
h & i & j & k & l
\end{array}\right) .
$$

Thus, if we call $J_{m}$ the integral obtained by applying $\tau^{m}$ to $J_{0}$, we have

$$
\begin{aligned}
& J_{0}=I(h, i, j, k, l), \\
& J_{1}=I(i, j, k, l, h), \\
& J_{2}=I(j, k, l, h, i), \\
& J_{3}=I(k, l, h, i, j), \\
& J_{4}=I(l, h, i, j, k) .
\end{aligned}
$$

Similarly, by applying to $I(h, i, j, k, l)$ the transformation

$$
\sigma:\left\{\begin{array}{l}
\xi=y, \\
\eta=x,
\end{array}\right.
$$

i.e. by interchanging the variables $x, y$ in the integral, we get $I(k, j, i, h, l)$. Hence with the action of $\sigma$ on $I(h, i, j, k, l)$ we associate the permutation

$$
\boldsymbol{\sigma}=\left(\begin{array}{lll}
h & k
\end{array}\right)\left(\begin{array}{ll}
i & j
\end{array}\right)
$$

that interchanges $h$ with $k$ and $i$ with $j$. Thus $\boldsymbol{\sigma}$ changes the diagram (2.2) by the symmetry about its vertical midline. Therefore the permutation group $\langle\boldsymbol{\tau}, \boldsymbol{\sigma}\rangle$ generated by $\boldsymbol{\tau}$ and $\boldsymbol{\sigma}$ is isomorphic to the dihedral group $\mathcal{D}_{5}$ of order 10 , and the value of $I(h, i, j, k, l)$ is invariant under the action of $\langle\boldsymbol{\tau}, \boldsymbol{\sigma}\rangle$.

Let now

$$
d_{n}=\text { l.c.m. }\{1,2, \ldots, n\},
$$

and $d_{0}=1$ for completeness. Our aim is to show that for any non-negative integers $h, i, j, k, l$ we have

$$
I(h, i, j, k, l)=a-b \zeta(2)
$$

with $a \in \mathbb{Q}, b \in \mathbb{Z}$, and to find two non-negative integers $M, N$, as small as possible, such that

$$
d_{M} d_{N} a \in \mathbb{Z},
$$

thus generalizing Hata's method [7]. The above $a$ and $b$ are the same for the ten integrals obtained by the action of $\langle\boldsymbol{\tau}, \boldsymbol{\sigma}\rangle$ on $I(h, i, j, k, l)$. Accordingly, $M$ and $N$ will be defined to be invariant under the actions of $\boldsymbol{\tau}$ and $\boldsymbol{\sigma}$ (see Theorem 2.2 below). 
TheOREM 2.1. Let $h, i, j, k, l$ be non-negative integers, and let

$$
\begin{aligned}
M_{0} & =\max \{k+l-i, l+h-j, i+j-l\}, \\
N_{0} & =\max \{j+k-h, \min \{k+l-i, l+h-j\}, h+i-k\} .
\end{aligned}
$$

Then the integral

$$
J_{0}=\int_{0}^{1} \int_{0}^{1} \frac{x^{h}(1-x)^{i} y^{k}(1-y)^{j}}{(1-x y)^{i+j-l}} \frac{d x d y}{1-x y}
$$

satisfies

$$
J_{0}=a-b \zeta(2)
$$

with $b \in \mathbb{Z}$ and $d_{M_{0}} d_{N_{0}} a \in \mathbb{Z}$.

Remark 2.1. Our assumption $h, i, j, k, l \geq 0$ obviously implies $M_{0}, N_{0} \geq 0$, even if some of the integers

$$
j+k-h, k+l-i, l+h-j, h+i-k, i+j-l
$$

are negative.

The proof of Theorem 2.1 is based on some arithmetical lemmas.

Lemma 2.1 (Beukers [1]). If $i+j-l \leq 0$, then Theorem 2.1 holds.

Proof. If $i+j-l<0$, since

$$
\int_{0}^{1} \int_{0}^{1} x^{r} y^{s} d x d y=\frac{1}{(r+1)(s+1)}
$$

for $r, s \geq 0$, we have $J_{0} \in \mathbb{Q}, d_{k+l-i} d_{l+h-j} J_{0} \in \mathbb{Z}$, and

$$
M_{0}=\max \{k+l-i, l+h-j\}, \quad N_{0} \geq \min \{k+l-i, l+h-j\} .
$$

If $i+j-l=0$, by Lemma 1.1 in [7] we have $J_{0}=a-b \zeta(2)$ with $b \in \mathbb{Z}$ and $d_{M^{\prime}} d_{N^{\prime}} a \in \mathbb{Z}$, where

$$
M^{\prime}=\max \{h+i, j+k\}=M_{0}
$$

and

$$
\begin{aligned}
N^{\prime} & =\min \{\max \{h+i, j+k-h\}, \max \{j+k, h+i-k\}\} \\
& =\max \{j+k-h, \min \{j+k, h+i\}, h+i-k\}=N_{0} .
\end{aligned}
$$

Lemma 2.2. If $j=k=0$, then Theorem 2.1 holds.

Proof. By (2.3) we have

$$
J_{0}=I(h, i, 0,0, l)=J_{2}=I(0,0, l, h, i),
$$

and

$$
M_{0}=\max \{l+h, i-l\}, \quad N_{0}=\max \{l-i, h+i\} .
$$


Denoting by $M_{2}$ and $N_{2}$ the corresponding integers for the integral $J_{2}$, i.e. the integers defined by (2.4) with $h, i, j, k, l$ replaced by $j, k, l, h, i$ respectively, we have in the present case

$$
M_{2}=\max \{h+i, l-i\}=N_{0}, \quad N_{2}=\max \{l+h, i-l\}=M_{0} .
$$

Hence the conclusion follows by applying Lemma 2.1 to $J_{0}$ if $i-l \leq 0$, or to $J_{2}$ if $i-l \geq 0$.

Lemma 2.3. If $k=l=0$, then Theorem 2.1 holds.

Proof. Again by (2.3) we have

$$
J_{0}=I(h, i, j, 0,0)=J_{1}=I(i, j, 0,0, h),
$$

and

$$
M_{0}=\max \{h-j, i+j\}, \quad N_{0}=\max \{j-h, h+i\} .
$$

Denoting by $M_{1}, N_{1}$ the integers defined by (2.4) with $h, i, j, k, l$ replaced by $i, j, k, l, h$ respectively, we now find

$$
M_{1}=\max \{h+i, j-h\}=N_{0}, \quad N_{1}=\max \{h-j, i+j\}=M_{0} .
$$

Hence the conclusion follows by applying Lemma 2.2 to $J_{1}$.

Lemma 2.4. If $i=k=0$, then Theorem 2.1 holds.

Proof. If $h=0$, we apply Lemma 2.2 after interchanging $x$ and $y$ in $J_{0}$. If $l=0$, we apply Lemma 2.3. If $j-l \leq 0$, we apply Lemma 2.1. Hence we may assume

$$
\min \{h, l, j-l\}>0 .
$$

Then we have the decomposition

$$
\begin{aligned}
J_{0} & =\int_{0}^{1} \int_{0}^{1} \frac{x^{h}(1-y)^{j}}{(1-x y)^{j-l}} \frac{d x d y}{1-x y} \\
& =\int_{0}^{1} \int_{0}^{1}\left(\frac{x^{h}(1-y)^{j-1}}{(1-x y)^{j-l}}-\frac{x^{h-1}(1-y)^{j-1}}{(1-x y)^{j-l}}+\frac{x^{h-1}(1-y)^{j-1}}{(1-x y)^{j-l-1}}\right) \frac{d x d y}{1-x y} \\
& =I(h, 0, j-1,0, l-1)-I(h-1,0, j-1,0, l-1)+I(h-1,0, j-1,0, l) \\
& =J_{0}^{(1)}-J_{0}^{(2)}+J_{0}^{(3)}
\end{aligned}
$$

say, with $J_{0}^{(r)}=I\left(h_{r}, 0, j_{r}, 0, l_{r}\right)$. For each $r, 1 \leq r \leq 3$, at least one of the integers $h_{r}, l_{r}, j_{r}-l_{r}$ is smaller than the corresponding integer associated with $J_{0}$, while none is larger. Moreover, if we denote by $M_{0}^{(r)}, N_{0}^{(r)}$ the integers defined by (2.4) with $h, i, j, k, l$ replaced by $h_{r}, 0, j_{r}, 0, l_{r}$ respectively, we see that $M_{0}^{(r)}, N_{0}^{(r)}$ do not exceed the corresponding integers $M_{0}, N_{0}$ for $J_{0}$. By iterating the above decomposition for each integral $J_{0}^{(r)}$ satisfying 
(2.5), i.e. such that $\min \left\{h_{r}, l_{r}, j_{r}-l_{r}\right\}>0$, we express $J_{0}$ as a linear combination with integer coefficients of finitely many integrals $J_{0}^{(s)}=a_{s}-b_{s} \zeta(2)$ satisfying $b_{s} \in \mathbb{Z}$ and $d_{M_{0}} d_{N_{0}} a_{s} \in \mathbb{Z}$.

Lemma 2.5. If $k=0$, then Theorem 2.1 holds.

Proof. If $i=0$ or $j=0$ or $l=0$, we apply Lemmas $2.4,2.2$ or 2.3 respectively. If $i+j-l \leq 0$, we apply Lemma 2.1. Hence we may assume

$$
\min \{i, j, l, i+j-l\}>0 .
$$

Then we iterate the decomposition

$$
\begin{aligned}
J_{0}= & \int_{0}^{1} \int_{0}^{1} \frac{x^{h}(1-x)^{i}(1-y)^{j}}{(1-x y)^{i+j-l}} \frac{d x d y}{1-x y} \\
= & \int_{0}^{1} \int_{0}^{1}\left(\frac{x^{h}(1-x)^{i}(1-y)^{j-1}}{(1-x y)^{i+j-l}}+\frac{x^{h}(1-x)^{i-1}(1-y)^{j}}{(1-x y)^{i+j-l}}\right. \\
& \left.-\frac{x^{h}(1-x)^{i-1}(1-y)^{j-1}}{(1-x y)^{i+j-l-1}}\right) \frac{d x d y}{1-x y} \\
= & I(h, i, j-1,0, l-1)+I(h, i-1, j, 0, l-1)-I(h, i-1, j-1,0, l-1)
\end{aligned}
$$

for each integral satisfying (2.6), and we conclude as in the proof of Lemma 2.4 .

Proof of Theorem 2.1. If $h=0$ or $k=0$ we apply Lemma 2.5, possibly after interchanging $x$ and $y$. If $i+j-l \leq 0$ we apply Lemma 2.1. Hence we may assume

$$
\min \{h, k, i+j-l\}>0 .
$$

Then we iterate the decomposition

$$
\begin{aligned}
J_{0} & =\int_{0}^{1} \int_{0}^{1} \frac{x^{h}(1-x)^{i} y^{k}(1-y)^{j}}{(1-x y)^{i+j-l}} \frac{d x d y}{1-x y} \\
& =\int_{0}^{1} \int_{0}^{1}\left(\frac{x^{h-1}(1-x)^{i} y^{k-1}(1-y)^{j}}{(1-x y)^{i+j-l}}-\frac{x^{h-1}(1-x)^{i} y^{k-1}(1-y)^{j}}{(1-x y)^{i+j-l-1}}\right) \frac{d x d y}{1-x y} \\
& =I(h-1, i, j, k-1, l)-I(h-1, i, j, k-1, l+1)
\end{aligned}
$$

for each integral satisfying (2.7), and we conclude as in the proof of Lemma 2.4 .

The integers $M_{0}, N_{0}$ defined by (2.4) are invariant under the action of $\boldsymbol{\sigma}$, but not of $\boldsymbol{\tau}$. For each $m, 0 \leq m \leq 4$, let $M_{m}, N_{m}$ be the corresponding integers for the integral $J_{m}$ in (2.3), i.e. those defined by (2.4) with $h, i, j$, 
$k, l$ replaced by

$$
\boldsymbol{\tau}^{m}(h), \boldsymbol{\tau}^{m}(i), \boldsymbol{\tau}^{m}(j), \boldsymbol{\tau}^{m}(k), \boldsymbol{\tau}^{m}(l)
$$

respectively, where $\boldsymbol{\tau}$ is the permutation (2.2). Since $J_{0}=\ldots=J_{4}$, for any $m$ and $q$ with $0 \leq m \leq 4,0 \leq q \leq 4$, the pair $M_{m}, N_{m}$ is admissible for $J_{q}$. In other words, we have $J_{q}=a-b \zeta(2)$ with $b \in \mathbb{Z}$ and $d_{M_{m}} d_{N_{m}} a \in \mathbb{Z}$. Thus we seek the best pairs among $M_{m}, N_{m}(0 \leq m \leq 4)$, i.e. those for which $M_{m}+N_{m}$ is minimal.

We let $\boldsymbol{\tau}$ and $\boldsymbol{\sigma}$ act on the five integers

$$
j+k-h, k+l-i, l+h-j, h+i-k, i+j-l
$$

by defining

$$
\begin{gathered}
\boldsymbol{\tau}(j+k-h)=\boldsymbol{\tau}(j)+\boldsymbol{\tau}(k)-\boldsymbol{\tau}(h)=k+l-i, \\
\boldsymbol{\sigma}(j+k-h)=\boldsymbol{\sigma}(j)+\boldsymbol{\sigma}(k)-\boldsymbol{\sigma}(h)=h+i-k,
\end{gathered}
$$

and so on. Hence the cyclic permutation

$$
(j+k-h k+l-i l+h-j h+i-k i+j-l)
$$

is also called $\tau$, and similarly

$$
(j+k-h h+i-k)(k+l-i l+h-j)
$$

is called $\boldsymbol{\sigma}$. We define

$$
\begin{aligned}
M & =\max \{j+k-h, k+l-i, l+h-j, h+i-k, i+j-l\}, \\
N & =\max \left\{\boldsymbol{\tau}(M), \min \left\{\boldsymbol{\tau}^{2}(M), \boldsymbol{\tau}^{3}(M)\right\}, \boldsymbol{\tau}^{4}(M)\right\} .
\end{aligned}
$$

If two (or more) of the five integers (2.8) are maximal, the definition of $N$, a priori, may depend on the choice of the maximal integer $M$ among (2.8). However, the proof of Theorem 2.2 will show that $N$ is well defined, for if $M$ and $M^{\prime}$ are both maximal, then the $N$ above and

$$
N^{\prime}=\max \left\{\boldsymbol{\tau}\left(M^{\prime}\right), \min \left\{\boldsymbol{\tau}^{2}\left(M^{\prime}\right), \boldsymbol{\tau}^{3}\left(M^{\prime}\right)\right\}, \boldsymbol{\tau}^{4}\left(M^{\prime}\right)\right\}
$$

are equal. Moreover, the $M, N$ defined by (2.9) are clearly invariant under the actions of $\boldsymbol{\tau}$ and $\boldsymbol{\sigma}$.

Theorem 2.2. Let $h, i, j, k, l$ be non-negative integers, let the integrals $J_{q}(0 \leq q \leq 4)$ be as in (2.3), and let $M, N$ be defined by (2.9). For any $q$ we have

$$
J_{q}=a-b \zeta(2)
$$

with $b \in \mathbb{Z}, d_{M} d_{N} a \in \mathbb{Z}$, and $M, N$ is the best among the (unordered) pairs $M_{m}, N_{m}(0 \leq m \leq 4)$.

Proof. We shall show that two cases may occur: either the five pairs $M_{m}, N_{m}$ are all equal (up to the interchange of $M_{m}$ and $N_{m}$ ) to the pair $M, N$, or three of them are equal to $M, N$ and the remaining two are equal 
to a pair worse than $M, N$. Thus in either case $M, N$ is the best pair for each $J_{q}$.

We may assume with no loss of generality that $M=i+j-l$. For otherwise we should replace $J_{0}$ with the integral $J_{r}$ such that $M=\tau^{r}(i+j-l)$, and accordingly each $J_{q}$ with the integral $J_{s}$ such that $s \equiv r+q(\bmod 5)$.

In each of the five pairs $M_{m}, N_{m}$ the maximal integer $i+j-l$ occurs either in the expression for $M_{m}$ or for $N_{m}$, so that one element of the best pair must be $M=i+j-l$. To find the other, we consider the different possibilities for the maximum of the remaining four integers. Note that the cases

$$
\max \{j+k-h, k+l-i, l+h-j, h+i-k\}=k+l-i
$$

and

$$
\max \{j+k-h, k+l-i, l+h-j, h+i-k\}=l+h-j
$$

are changed into each other by $\boldsymbol{\sigma}$, so that the same discussion applies to both, and similarly for the cases

$$
\max \{j+k-h, k+l-i, l+h-j, h+i-k\}=j+k-h
$$

and

$$
\max \{j+k-h, k+l-i, l+h-j, h+i-k\}=h+i-k .
$$

Therefore, up to the action of $\boldsymbol{\sigma}$, we have only twelve distinct possibilities for the ordering of the integers (2.8):

$$
\begin{gathered}
j+k-h \leq l+h-j \leq h+i-k \leq k+l-i \leq i+j-l, \\
l+h-j \leq j+k-h \leq h+i-k \leq k+l-i \leq i+j-l, \\
j+k-h \leq h+i-k \leq l+h-j \leq k+l-i \leq i+j-l, \\
h+i-k \leq j+k-h \leq l+h-j \leq k+l-i \leq i+j-l, \\
l+h-j \leq h+i-k \leq j+k-h \leq k+l-i \leq i+j-l, \\
h+i-k \leq l+h-j \leq j+k-h \leq k+l-i \leq i+j-l,
\end{gathered}
$$

and

$$
\begin{array}{r}
k+l-i \leq l+h-j \leq h+i-k \leq j+k-h \leq i+j-l, \\
l+h-j \leq k+l-i \leq h+i-k \leq j+k-h \leq i+j-l, \\
k+l-i \leq h+i-k \leq l+h-j \leq j+k-h \leq i+j-l, \\
h+i-k \leq k+l-i \leq l+h-j \leq j+k-h \leq i+j-l, \\
l+h-j \leq h+i-k \leq k+l-i \leq j+k-h \leq i+j-l, \\
h+i-k \leq l+h-j \leq k+l-i \leq j+k-h \leq i+j-l .
\end{array}
$$

In the five pairs $M_{m}, N_{m}$ the smaller of $M_{m}$ and $N_{m}$ is $h+i-k$ three times and $k+l-i$ twice if we are in one of the cases $(2.10) ; l+h-j$ three times 
and $k+l-i$ twice in (2.11); $j+k-h$ three times and $k+l-i$ twice in (2.12). In any of the cases (2.13) we find $j+k-h$ all the five times. Hence in any of the above cases the best choice for the smaller of $M_{m}$ and $N_{m}$ $(m=0, \ldots, 4)$ is

$$
N=\max \{j+k-h, \min \{k+l-i, l+h-j\}, h+i-k\} .
$$

This proves Theorem 2.2.

Under the assumptions of Theorems 2.1 or 2.2 , the integer $b$ can be expressed as a double contour integral.

Lemma 2.6. Let $h, i, j, k, l$ be non-negative integers. If

$$
J_{0}=\int_{0}^{1} \int_{0}^{1} \frac{x^{h}(1-x)^{i} y^{k}(1-y)^{j}}{(1-x y)^{i+j-l}} \frac{d x d y}{1-x y}=a-b \zeta(2)
$$

with $a \in \mathbb{Q}$ and $b \in \mathbb{Z}$, then, for any $\varrho_{1}, \varrho_{2}>0$,

$$
b=-\frac{1}{4 \pi^{2}} \int_{C} \int_{C_{x}} \frac{x^{h}(1-x)^{i} y^{k}(1-y)^{j}}{(1-x y)^{i+j-l}} \frac{d x d y}{1-x y},
$$

where $C=\left\{x \in \mathbb{C}:|x|=\varrho_{1}\right\}$ and $C_{x}=\left\{y \in \mathbb{C}:|y-1 / x|=\varrho_{2}\right\}$.

Proof. As in the proof of Theorem 2.1, we can express $J_{0}$ as a linear combination with integer coefficients of integrals having either $i+j-l \leq 0$, or, possibly after interchanging $x$ and $y, k=0$. If $k=0$, by the proofs of Lemmas 2.5 and 2.4 we express the integral as a linear combination with integer coefficients of integrals either having $i+j-l \leq 0$, or such that at least two consecutive integers among $h, i, j, k, l$ vanish. In the latter case, by applying a suitable power of the transformation $\tau$ we may assume $j=$ $k=0$, and then the proof of Lemma 2.2 shows that the integral either has $i+j-l \leq 0$ or can be transformed into one having $i+j-l \leq 0$. If $i+j-l<0$, the integral is a rational number. Therefore

$$
J_{0}=\sum_{t=1}^{T} \beta_{t} J^{(t)}+(\text { rational number }),
$$

where $\beta_{t} \in \mathbb{Z}$ and

$$
J^{(t)}=\int_{0}^{1} \int_{0}^{1} x^{h_{t}}(1-x)^{i_{t}} y^{k_{t}}(1-y)^{j_{t}} \frac{d x d y}{1-x y} .
$$

Thus, if we put

$$
J^{(t)}=a^{(t)}-b^{(t)} \zeta(2)
$$


with $a^{(t)} \in \mathbb{Q}$ and $b^{(t)} \in \mathbb{Z}$, we get

$$
b=\sum_{t=1}^{T} \beta_{t} b^{(t)} .
$$

We now apply to the double contour integral

$$
\widetilde{J}_{0}=-\frac{1}{4 \pi^{2}} \int_{C} \int_{C_{x}} \frac{x^{h}(1-x)^{i} y^{k}(1-y)^{j}}{(1-x y)^{i+j-l}} \frac{d x d y}{1-x y}
$$

the same linear decompositions and the same transformations used for $J_{0}$. Since $\tau$ changes

$$
\int_{C} \int_{C_{x}} \frac{x^{h}(1-x)^{i} y^{k}(1-y)^{j}}{(1-x y)^{i+j-l}} \frac{d x d y}{1-x y}
$$

into

which clearly equals

$$
\int_{|y|=\varrho_{1} \varrho_{2}} \int_{|x-1 / y|=1 / \varrho_{2}} \frac{x^{i}(1-x)^{j} y^{l}(1-y)^{k}}{(1-x y)^{j+k-h}} \frac{d x d y}{1-x y},
$$

$$
\int_{|x|=\varrho_{1}^{\prime}} \int_{|y-1 / x|=\varrho_{2}^{\prime}} \frac{x^{i}(1-x)^{j} y^{l}(1-y)^{k}}{(1-x y)^{j+k-h}} \frac{d x d y}{1-x y}
$$

for any $\varrho_{1}, \varrho_{2}, \varrho_{1}^{\prime}, \varrho_{2}^{\prime}>0$, and since (2.17) vanishes if $i+j-l<0$, we obtain

$$
\widetilde{J}_{0}=\sum_{t=1}^{T} \beta_{t} \widetilde{J}^{(t)},
$$

with the same $\beta_{t} \in \mathbb{Z}$ as above and with

$$
\widetilde{J}^{(t)}=-\frac{1}{4 \pi^{2}} \int_{C} \int_{C_{x}} x^{h_{t}}(1-x)^{i_{t}} y^{k_{t}}(1-y)^{j_{t}} \frac{d x d y}{1-x y} .
$$

Let now $P(x, y)=\sum_{r=0}^{\delta_{1}} \sum_{s=0}^{\delta_{2}} \alpha_{r s} x^{r} y^{s}$ be any polynomial with $\alpha_{r s} \in \mathbb{Z}$. Then (see [7], Lemma 1.1)

$$
\int_{0}^{1} \int_{0}^{1} P(x, y) \frac{d x d y}{1-x y}=\sum_{r=0}^{\delta_{1}} \sum_{s=0}^{\delta_{2}} \alpha_{r s} \int_{0}^{1} \int_{0}^{1} x^{r} y^{s} \frac{d x d y}{1-x y}=U-V \zeta(2),
$$

with $U \in \mathbb{Q}$ and (denoting here $i=\sqrt{-1}$ )

$$
\begin{aligned}
-V & =\sum_{r=0}^{\min \left\{\delta_{1}, \delta_{2}\right\}} \alpha_{r r}=\sum_{r=0}^{\delta_{1}} \sum_{s=0}^{\delta_{2}} \alpha_{r s} \frac{1}{2 \pi i} \int_{|z|=\varrho_{1}} z^{r-s-1} d z \\
& =\frac{1}{2 \pi i} \int_{|z|=\varrho_{1}} P(z, 1 / z) \frac{d z}{z} .
\end{aligned}
$$


For any $z \neq 0$ we have

$$
-\frac{1}{2 \pi i} \int_{|y-1 / z|=\varrho_{2}} \frac{P(z, y)}{1-z y} d y=\frac{1}{z} \frac{1}{2 \pi i} \int_{|y-1 / z|=\varrho_{2}} \frac{P(z, y)}{y-1 / z} d y=\frac{1}{z} P(z, 1 / z) .
$$

Hence

$$
\begin{aligned}
V & =-\frac{1}{2 \pi i} \int_{|z|=\varrho_{1}}\left(-\frac{1}{2 \pi i} \int_{|y-1 / z|=\varrho_{2}} \frac{P(z, y)}{1-z y} d y\right) d z \\
& =-\frac{1}{4 \pi^{2}} \int_{|z|=\varrho_{1}} \int_{|y-1 / z|=\varrho_{2}} \frac{P(z, y)}{1-z y} d y d z .
\end{aligned}
$$

Taking $P(x, y)=x^{h_{t}}(1-x)^{i_{t}} y^{k_{t}}(1-y)^{j_{t}}$, from (2.14), (2.15) and (2.19) we get

$$
b^{(t)}=-\frac{1}{4 \pi^{2}} \int_{|x|=\varrho_{1}} \int_{|y-1 / x|=\varrho_{2}} x^{h_{t}}(1-x)^{i_{t}} y^{k_{t}}(1-y)^{j_{t}} \frac{d x d y}{1-x y}=\widetilde{J}^{(t)} .
$$

Therefore, by (2.16) and (2.18),

$$
b=\sum_{t=1}^{T} \beta_{t} b^{(t)}=\sum_{t=1}^{T} \beta_{t} \widetilde{J}^{(t)}=\widetilde{J}_{0} .
$$

3. The hypergeometric permutation. Let $\alpha, \beta, \gamma$ be complex parameters, $\gamma \neq 0,-1,-2, \ldots$, and $y$ a complex variable satisfying $|y|<1$. The Gauss hypergeometric function $F(\alpha, \beta ; \gamma ; y)={ }_{2} F_{1}(\alpha, \beta ; \gamma ; y)$ is defined by

$$
F(\alpha, \beta ; \gamma ; y)=\sum_{n=0}^{\infty} \frac{(\alpha)_{n}(\beta)_{n}}{(\gamma)_{n}} \cdot \frac{y^{n}}{n !}
$$

where $(\alpha)_{0}=1,(\alpha)_{n}=\alpha(\alpha+1) \ldots(\alpha+n-1) \quad(n=1,2, \ldots)$, and similarly for $(\beta)_{n}$ and $(\gamma)_{n}$. By Euler's integral representation we have, for $\operatorname{Re} \gamma>$ $\operatorname{Re} \beta>0$,

$$
F(\alpha, \beta ; \gamma ; y)=\frac{\Gamma(\gamma)}{\Gamma(\beta) \Gamma(\gamma-\beta)} \int_{0}^{1} \frac{x^{\beta-1}(1-x)^{\gamma-\beta-1}}{(1-x y)^{\alpha}} d x
$$

([4], p. 59), and this gives the analytic continuation of $F(\alpha, \beta ; \gamma ; y)$. Since, by $(3.1)$,

$$
F(\alpha, \beta ; \gamma ; y)=F(\beta, \alpha ; \gamma ; y),
$$

if $\operatorname{Re} \gamma>\max \{\operatorname{Re} \alpha, \operatorname{Re} \beta\}$ and $\min \{\operatorname{Re} \alpha, \operatorname{Re} \beta\}>0$ we obtain 


$$
\begin{aligned}
& \frac{1}{\Gamma(\beta) \Gamma(\gamma-\beta)} \int_{0}^{1} \frac{x^{\beta-1}(1-x)^{\gamma-\beta-1}}{(1-x y)^{\alpha}} d x \\
&=\frac{1}{\Gamma(\alpha) \Gamma(\gamma-\alpha)} \int_{0}^{1} \frac{x^{\alpha-1}(1-x)^{\gamma-\alpha-1}}{(1-x y)^{\beta}} d x
\end{aligned}
$$

Here and in the sequel we choose five non-negative integers $h, i, j, k, l$ such that $j+k-h, k+l-i, l+h-j, h+i-k, i+j-l$ are also non-negative. Taking in (3.2)

$$
\alpha=i+j-l+1, \quad \beta=h+1, \quad \gamma=h+i+2,
$$

we get

$$
\begin{aligned}
\frac{1}{h ! i !} \int_{0}^{1} \frac{x^{h}(1-x)^{i}}{(1-x y)^{i+j-l+1}} & d x \\
& =\frac{1}{(i+j-l) !(l+h-j) !} \int_{0}^{1} \frac{x^{i+j-l}(1-x)^{l+h-j}}{(1-x y)^{h+1}} d x .
\end{aligned}
$$

Multiplying by $y^{k}(1-y)^{j}$ and integrating in $0 \leq y \leq 1$ we obtain, by (2.1),

$$
\begin{aligned}
& \frac{1}{h ! i !} I(h, i, j, k, l) \\
& \quad=\frac{1}{(i+j-l) !(l+h-j) !} I(i+j-l, l+h-j, j, k, l) .
\end{aligned}
$$

Dividing (3.3) by $j ! k ! l$ ! we have

$$
\frac{I(h, i, j, k, l)}{h ! i ! j ! k ! l !}=\frac{I(i+j-l, l+h-j, j, k, l)}{(i+j-l) !(l+h-j) ! j ! k ! l !} .
$$

Let $\varphi$ be the hypergeometric integral transformation acting on

$$
\frac{I(h, i, j, k, l)}{h ! i ! j ! k ! l !}
$$

as is described above. It is natural to associate with the action of $\varphi$ on (3.5) a permutation $\varphi$ of the integers $h, i, j, k, l, j+k-h, k+l-i, l+h-j$, $h+i-k, i+j-l$ which we define to be the following product of transpositions (i.e. 2-cycles):

$$
\boldsymbol{\varphi}=(h i+j-l)(i l+h-j)(j+k-h k+l-i) .
$$

Note that

$$
\boldsymbol{\varphi}(j+k-h)=k+l-i=j+k-(i+j-l)=\boldsymbol{\varphi}(j)+\boldsymbol{\varphi}(k)-\boldsymbol{\varphi}(h),
$$

and similarly $\varphi(k+l-i)=\varphi(k)+\varphi(l)-\varphi(i)$, etc. In accordance with 
Section 2, we call $\boldsymbol{\tau}$ and $\boldsymbol{\sigma}$ the permutations

$$
\begin{aligned}
& \boldsymbol{\tau}=\left(\begin{array}{lllll}
h & i & j & k & l
\end{array}\right)(j+k-h k+l-i l+h-j h+i-k i+j-l), \\
& \boldsymbol{\sigma}=\left(\begin{array}{lll}
h & k
\end{array}\right)\left(\begin{array}{lll}
i & j
\end{array}\right)(j+k-h h+i-k)(k+l-i l+h-j) .
\end{aligned}
$$

We are interested in the structure of the permutation group

$$
\boldsymbol{\Phi}=\langle\boldsymbol{\varphi}, \boldsymbol{\tau}, \boldsymbol{\sigma}\rangle
$$

generated by $\boldsymbol{\varphi}, \boldsymbol{\tau}$ and $\boldsymbol{\sigma}$. We have already remarked that the subgroup

$$
\mathbf{T}=\langle\boldsymbol{\tau}, \boldsymbol{\sigma}\rangle
$$

is isomorphic to the dihedral group $\mathcal{D}_{5}$ of order 10 , and it is easy to check that $\langle\boldsymbol{\varphi}, \boldsymbol{\sigma}\rangle$ is isomorphic to the dihedral group $\mathcal{D}_{6}$ of order 12 . Following a remark of Dixon [2], we note that $\boldsymbol{\varphi}, \boldsymbol{\tau}$ and $\boldsymbol{\sigma}$ can be viewed as three permutations of five integers only, i.e. of the five sums

$$
h+i, i+j, j+k, k+l, l+h,
$$

by defining $\varphi(h+i)=\varphi(h)+\varphi(i)$, etc. In fact, we have the following decompositions into cycles:

$$
\begin{aligned}
& \boldsymbol{\varphi}=(i+j l+h), \\
& \boldsymbol{\tau}=(h+i i+j j+k k+l l+h), \\
& \boldsymbol{\sigma}=(h+i j+k)(k+l l+h) .
\end{aligned}
$$

Since the symmetric group $\mathcal{S}_{5}$ of the $5 !=120$ permutations of five elements is generated by a cyclic permutation of the five elements and a transposition, we see that

$$
\boldsymbol{\Phi}=\langle\boldsymbol{\varphi}, \boldsymbol{\tau}, \boldsymbol{\sigma}\rangle=\langle\boldsymbol{\varphi}, \boldsymbol{\tau}\rangle
$$

is isomorphic to $\mathcal{S}_{5}$. Moreover, the value of (3.5) is invariant under the action of $\boldsymbol{\Phi}$, whence (3.5) is a symmetric function of the sums $h+i, i+j, j+k$, $k+l, l+h$.

Since $|\mathbf{\Phi}|=120$ and $|\mathbf{T}|=10$, there are 12 left cosets of $\mathbf{T}$ in $\mathbf{\Phi}$. Each left coset can be characterized in terms of the factorials occurring in the corresponding transformation formulae for $I(h, i, j, k, l)$ such as (3.3). To see this, note that from our definitions it follows that if we apply to (3.5) any product $\chi$ of integral transformations $\varphi, \tau$ and $\sigma$, we obtain

$$
\frac{I(\chi(h), \chi(i), \chi(j), \chi(k), \chi(l))}{\chi(h) ! \chi(i) ! \chi(j) ! \chi(k) ! \chi(l) !},
$$

where $\chi$ is the corresponding product of permutations $\varphi, \tau$ and $\boldsymbol{\sigma}$ in reverse order. In other words, the above mapping $\chi \mapsto \chi$ between the groups $\Phi=$ $\langle\varphi, \tau, \sigma\rangle$ and $\boldsymbol{\Phi}=\langle\boldsymbol{\varphi}, \boldsymbol{\tau}, \boldsymbol{\sigma}\rangle$ is an anti-isomorphism. For instance, if we apply $\tau \varphi$ (i.e. first $\varphi$ and then $\tau$ ) to (3.5) we get

$$
\frac{I(h, i, j, k, l)}{h ! i ! j ! k ! l !}=\frac{I(i+j-l, l+h-j, j, k, l)}{(i+j-l) !(l+h-j) ! j ! k ! l !}=\frac{I(l+h-j, j, k, l, i+j-l)}{(l+h-j) ! j ! k ! l !(i+j-l) !},
$$


and if we apply $\boldsymbol{\varphi} \boldsymbol{\tau}$ (first $\boldsymbol{\tau}$ and then $\boldsymbol{\varphi}$ ) to $(h, i, j, k, l)$ we find

$$
(h, i, j, k, l) \mapsto(i, j, k, l, h) \mapsto(l+h-j, j, k, l, i+j-l) .
$$

Therefore, if for any $\varrho \in \mathbf{T}=\langle\boldsymbol{\tau}, \boldsymbol{\sigma}\rangle$ we apply $\boldsymbol{\varphi} \varrho$ to $(h, i, j, k, l)$, we change both the numerator and the denominator of the right side of (3.4) by a suitable permutation of $i+j-l, l+h-j, j, k, l$. If the numerator is changed into an integral $\widetilde{I}$, we obtain

$$
\begin{aligned}
I(h, i, j, k, l) & =\frac{h ! i !}{(i+j-l) !(l+h-j) !} I(i+j-l, l+h-j, j, k, l) \\
& =\frac{h ! i !}{(i+j-l) !(l+h-j) !} \widetilde{I} .
\end{aligned}
$$

Thus we find the same factor

$$
\frac{h ! i !}{(i+j-l) !(l+h-j) !}
$$

for each of the ten elements of the left coset $\varphi \mathbf{T}$. Plainly the same argument applies to every left coset of $\mathbf{T}$ in $\boldsymbol{\Phi}$.

Since every element of $\boldsymbol{\Phi}=\langle\boldsymbol{\varphi}, \boldsymbol{\tau}\rangle$ is a suitable product of permutations each equal to $\varphi$ or to $\tau$, since the value of (3.5) is invariant under the action of $\boldsymbol{\Phi}$ and moreover $h+i=(i+j-l)+(l+h-j)$, we see that in the transformation formulae for $I(h, i, j, k, l)$ each factor of the type (3.6) corresponding to a left coset of $\mathbf{T}$ in $\mathbf{\Phi}$ is a quotient of factorials satisfying the following properties:

(i) The numerator and the denominator are products of the same number of factorials.

(ii) The integers occurring in the numerator belong to the set $\{h, i, j, k, l\}$ and the integers in the denominator belong to $\{j+k-h, k+l-i, l+h-$ $j, h+i-k, i+j-l\}$.

(iii) The sum of the integers in the numerator equals the sum of the integers in the denominator.

It is easy to see that the following elements of $\boldsymbol{\Phi}$ :

$$
\begin{array}{r}
\iota, \varphi, \tau \varphi, \tau^{2} \varphi, \tau^{3} \varphi, \tau^{4} \varphi, \varphi \tau \varphi, \varphi \tau^{2} \varphi, \varphi \tau^{3} \varphi \\
\varphi \tau^{4} \varphi, \tau^{2} \varphi \tau \varphi, \varphi \tau^{2} \varphi \tau \varphi
\end{array}
$$

where $\iota$ denotes the identity, yield distinct factors of the type (3.6), and hence are pairwise left-inequivalent $\bmod \mathbf{T}$, i.e. representatives of all the 12 left cosets of $\mathbf{T}$ in $\mathbf{\Phi}$.

Let the permutations (3.7) be denoted by $\chi_{1}, \chi_{2}, \ldots, \chi_{12}$ respectively. The corresponding transformation formulae for $I(h, i, j, k, l)$ are the following: 


$$
\begin{aligned}
& I(h, i, j, k, l) \\
& =I(h, i, j, k, l) \\
& =\frac{h ! i !}{(i+j-l) !(l+h-j) !} I(i+j-l, l+h-j, j, k, l) \\
& =\frac{i ! j !}{(j+k-h) !(h+i-k) !} I(j+k-h, h+i-k, k, l, h) \\
& =\frac{j ! k !}{(k+l-i) !(i+j-l) !} I(k+l-i, i+j-l, l, h, i) \\
& =\frac{k ! l !}{(l+h-j) !(j+k-h) !} I(l+h-j, j+k-h, h, i, j) \\
& =\frac{l ! h !}{(h+i-k) !(k+l-i) !} I(h+i-k, k+l-i, i, j, k) \\
& =\frac{h ! i ! j !}{(k+l-i) !(h+i-k) !(i+j-l) !} \\
& \times I(k+l-i, h+i-k, k, l, i+j-l) \\
& =\frac{i ! j ! k !}{(j+k-h) !(i+j-l) !(l+h-j) !} \\
& \times I(j+k-h, h, l, i+j-l, l+h-j) \\
& =\frac{k ! l ! h !}{(k+l-i) !(i+j-l) !(l+h-j) !} \\
& \times I(i, k+l-i, i+j-l, l+h-j, j) \\
& =\frac{l ! h ! i !}{(h+i-k) !(j+k-h) !(l+h-j) !} \\
& \times I(h+i-k, j+k-h, l+h-j, j, k) \\
& =\frac{j ! k ! l !}{(h+i-k) !(j+k-h) !(k+l-i) !} \\
& \times I(h+i-k, j+k-h, h, i, k+l-i) \\
& =\frac{h ! i ! j ! k ! l !}{(h+i-k) !(k+l-i) !(i+j-l) !(l+h-j) !(j+k-h) !} \\
& \times I(h+i-k, k+l-i, i+j-l, l+h-j, j+k-h) .
\end{aligned}
$$

Denote the integrals occurring in the above formulae by $I^{(1)}, \ldots, I^{(12)}$ respectively. Let $M^{(1)}, N^{(1)}$ be the integers $(2.9)$, associated with $I^{(1)}=$ $I(h, i, j, k, l)$, and for every $r=1, \ldots, 12$ let $M^{(r)}, N^{(r)}$ be the corresponding integers associated with $I^{(r)}$. Thus we have, for $1 \leq r \leq 12$,

$$
I^{(r)}=I\left(\chi_{r}(h), \chi_{r}(i), \chi_{r}(j), \chi_{r}(k), \chi_{r}(l)\right)
$$


and

$$
\begin{gathered}
M^{(r)}=\max \left\{\chi_{r}(j+k-h), \chi_{r}(k+l-i), \chi_{r}(l+h-j),\right. \\
\left.\chi_{r}(h+i-k), \chi_{r}(i+j-l)\right\}, \\
N^{(r)}=\max \left\{\boldsymbol{\tau}_{r}\left(M^{(r)}\right), \min \left\{\boldsymbol{\tau}_{r}^{2}\left(M^{(r)}\right), \boldsymbol{\tau}_{r}^{3}\left(M^{(r)}\right)\right\}, \boldsymbol{\tau}_{r}^{4}\left(M^{(r)}\right)\right\},
\end{gathered}
$$

where

$$
\boldsymbol{\tau}_{r}=\boldsymbol{\chi}_{r} \boldsymbol{\tau} \boldsymbol{\chi}_{r}^{-1}
$$

Re mark 3.1. As we have already noted, the natural mapping $\Phi \rightarrow \boldsymbol{\Phi}$ considered above is an anti-isomorphism. Hence, for any $\boldsymbol{\chi} \in \mathbf{\Phi}$ and $\varrho \in \mathbf{T}$, the integral

$$
I(\chi \varrho(h), \chi \varrho(i), \chi \varrho(j), \chi \varrho(k), \chi \varrho(l))
$$

is obtained by applying to

$$
I(\chi(h), \chi(i), \chi(j), \chi(k), \chi(l))
$$

the transformation $\varrho \in T=\langle\tau, \sigma\rangle$ corresponding to the permutation $\varrho$. Since the $M, N$ defined by (2.9) are the same for each of ten integrals equivalent under the action of the transformation group $T$, the same integers $M, N$ are associated with the integrals (3.8) and (3.9). Therefore, for every $r=$ $1, \ldots, 12$ the pair $M^{(r)}, N^{(r)}$ is associated with each of the ten integrals obtained by applying to $(h, i, j, k, l)$ the elements of the left coset $\chi_{r} \mathbf{T}$. Thus if for any given $\boldsymbol{\chi} \in \mathbf{\Phi}$ we replace $I^{(1)}=I(h, i, j, k, l)$ with

$$
I^{\prime(1)}=I\left(h^{\prime}, i^{\prime}, j^{\prime}, k^{\prime}, l^{\prime}\right)=I(\chi(h), \chi(i), \chi(j), \chi(k), \chi(l)),
$$

and accordingly each $I^{(r)}$ with

$$
I^{\prime(r)}=I\left(\chi_{r}^{\prime}\left(h^{\prime}\right), \chi_{r}^{\prime}\left(i^{\prime}\right), \chi_{r}^{\prime}\left(j^{\prime}\right), \chi_{r}^{\prime}\left(k^{\prime}\right), \chi_{r}^{\prime}\left(l^{\prime}\right)\right),
$$

where

$$
\chi_{r}^{\prime}=\chi \chi_{r} \chi^{-1}
$$

we have

$$
I^{\prime(r)}=I\left(\chi \chi_{r}(h), \chi \chi_{r}(i), \chi \chi_{r}(j), \chi \chi_{r}(k), \chi \chi_{r}(l)\right),
$$

and the permutations $\chi \chi_{1}, \ldots, \chi \chi_{12}$ are pairwise left-inequivalent mod $\mathbf{T}$. Hence the 12 pairs $M^{\prime(r)}, N^{\prime(r)}$ associated with $I^{\prime(r)}(r=1, \ldots, 12)$ are the pairs $M^{(r)}, N^{(r)}$ in a different order.

For the applications given in the next sections, we require the following

LEMmA 3.1. There exists $s, 1 \leq s \leq 12$, such that for every $r=1, \ldots, 12$ we have $M^{(r)} \leq M^{(s)}$ and $N^{(r)} \leq N^{(\bar{s})}$.

Pro of. Since

$$
M^{(1)}=\max \{j+k-h, k+l-i, l+h-j, h+i-k, i+j-l\}
$$


and

$$
M^{(12)}=\max \{k, i, l, j, h\},
$$

it is plain that

$$
\begin{aligned}
M & =\max _{1 \leq r \leq 12} M^{(r)} \\
& =\max \{h, i, j, k, l, j+k-h, k+l-i, l+h-j, h+i-k, i+j-l\} .
\end{aligned}
$$

By Remark 3.1 we may assume with no loss of generality that

$$
M=\max _{1 \leq r \leq 12} M^{(r)}=i+j-l,
$$

for otherwise we should replace $I^{(1)}=I(h, i, j, k, l)$ with $I(\chi(h), \chi(i), \chi(j)$, $\chi(k), \chi(l))$ for a permutation $\chi \in \Phi$ such that $M=\chi(i+j-l)$.

From the expressions for $M^{(1)}, \ldots, M^{(12)}$ we see that each of the integers $h, i, j, k, l, j+k-h, k+l-i, l+h-j, h+i-k, i+j-l$ occurs exactly in six among $M^{(1)}, \ldots, M^{(12)}$. Let $M^{\left(r_{1}\right)}, \ldots, M^{\left(r_{6}\right)}$ be those containing the maximal integer $i+j-l$ in their expressions, whence

$$
M=M^{\left(r_{1}\right)}=\ldots=M^{\left(r_{6}\right)}=i+j-l,
$$

and let

$$
N=\max \left\{N^{\left(r_{1}\right)}, \ldots, N^{\left(r_{6}\right)}\right\}
$$

(we actually have $\left\{r_{1}, \ldots, r_{6}\right\}=\{1,3,5,6,10,11\}$, but we do not need this information). In order to show that $M, N$ is the maximal pair $M^{(s)}, N^{(s)}$ we are seeking, we must prove that $N^{(r)} \leq N$ for any $r \neq r_{1}, \ldots, r_{6}$.

If $r=r_{m}, 1 \leq m \leq 6$, we know that

$$
N \geq N^{\left(r_{m}\right)} \geq \max \left\{\boldsymbol{\tau}_{r_{m}}(i+j-l), \boldsymbol{\tau}_{r_{m}}^{4}(i+j-l)\right\},
$$

where $\boldsymbol{\tau}_{r_{m}}(i+j-l)$ and $\boldsymbol{\tau}_{r_{m}}^{4}(i+j-l)$ are (cyclically) consecutive to $i+j-l$ on either side in the expression for $M^{\left(r_{m}\right)}$. If we pick the integers consecutive to $i+j-l$ in the expressions for $M^{\left(r_{1}\right)}, \ldots, M^{\left(r_{6}\right)}$, we see that

$$
j+k-h, h+i-k, h, i, j, k \leq N .
$$

Also, for any $r \neq r_{1}, \ldots, r_{6}$ the expression for $M^{(r)}$ contains exactly four among the integers

$$
j+k-h, h+i-k, h, i, j, k .
$$

If the integer $Q_{r}$ different from (3.11) in the expression for $M^{(r)}$ satisfies $Q_{r} \leq N$, then $N^{(r)} \leq M^{(r)} \leq N$; if $Q_{r}>N$ then $Q_{r}=M^{(r)}$ by (3.10), and

$$
N^{(r)}=\max \left\{\boldsymbol{\tau}_{r}\left(Q_{r}\right), \min \left\{\boldsymbol{\tau}_{r}^{2}\left(Q_{r}\right), \boldsymbol{\tau}_{r}^{3}\left(Q_{r}\right)\right\}, \boldsymbol{\tau}_{r}^{4}\left(Q_{r}\right)\right\}
$$

is the maximum of three integers among (3.11), whence $N^{(r)} \leq N$ by (3.10).

We conclude this section with two conjectures. 
Conjecture 3.1. Let $h, i, j, k, l$ and $h^{\prime}, i^{\prime}, j^{\prime}, k^{\prime}, l^{\prime}$ be any non-negative integers. If

$$
I(h, i, j, k, l)=I\left(h^{\prime}, i^{\prime}, j^{\prime}, k^{\prime}, l^{\prime}\right),
$$

then there exists a permutation $\varrho \in \mathbf{T}=\langle\boldsymbol{\tau}, \boldsymbol{\sigma}\rangle$ such that

$$
h^{\prime}=\varrho(h), \quad i^{\prime}=\varrho(i), \quad j^{\prime}=\varrho(j), \quad k^{\prime}=\varrho(k), \quad l^{\prime}=\varrho(l) .
$$

ConjeCture 3.2. Let $h, i, j, k, l$ and $h^{\prime}, i^{\prime}, j^{\prime}, k^{\prime}, l^{\prime}$ be any non-negative integers such that

$$
j+k-h, k+l-i, l+h-j, h+i-k, i+j-l
$$

and

$$
j^{\prime}+k^{\prime}-h^{\prime}, k^{\prime}+l^{\prime}-i^{\prime}, l^{\prime}+h^{\prime}-j^{\prime}, h^{\prime}+i^{\prime}-k^{\prime}, i^{\prime}+j^{\prime}-l^{\prime}
$$

are also non-negative. If

$$
\frac{I(h, i, j, k, l)}{I\left(h^{\prime}, i^{\prime}, j^{\prime}, k^{\prime}, l^{\prime}\right)} \in \mathbb{Q},
$$

then there exists a permutation $\boldsymbol{\chi} \in \mathbf{\Phi}=\langle\boldsymbol{\varphi}, \boldsymbol{\tau}, \boldsymbol{\sigma}\rangle$ such that

$$
h^{\prime}=\chi(h), \quad i^{\prime}=\chi(i), \quad j^{\prime}=\chi(j), \quad k^{\prime}=\chi(k), \quad l^{\prime}=\chi(l) .
$$

4. The $p$-adic valuation. Given five non-negative integers $h, i, j, k, l$ such that $j+k-h, k+l-i, l+h-j, h+i-k, i+j-l$ are also nonnegative, we consider again the integrals $I^{(1)}, \ldots, I^{(12)}$ obtained by applying to $(h, i, j, k, l)$ the permutations $(3.7)$, and for every $r=1, \ldots, 12$ the pair $M^{(r)}, N^{(r)}$ associated with $I^{(r)}$, as is described in Section 3. By Remark 3.1 we may and shall assume, with no loss of generality, that the maximal pair $M^{(s)}, N^{(s)}$ considered in Lemma 3.1 is obtained for $s=1$, for otherwise we should replace $I^{(1)}=I(h, i, j, k, l)$ with

$$
I^{\prime(1)}=I\left(h^{\prime}, i^{\prime}, j^{\prime}, k^{\prime}, l^{\prime}\right)=I^{(s)}=I\left(\chi_{s}(h), \chi_{s}(i), \chi_{s}(j), \chi_{s}(k), \chi_{s}(l)\right) .
$$

For $r=1, \ldots, 12$ and $n=1,2, \ldots$ we define

$$
I_{n}^{(r)}=I\left(\chi_{r}(h) n, \chi_{r}(i) n, \chi_{r}(j) n, \chi_{r}(k) n, \chi_{r}(l) n\right)=a_{n}^{(r)}-b_{n}^{(r)} \zeta(2),
$$

where $\chi_{r}$ is the $r$ th permutation in the list (3.7). By Theorem 2.2 we have $b_{n}^{(r)} \in \mathbb{Z}$ and $d_{M^{(r)} n} d_{N^{(r)} n} a_{n}^{(r)} \in \mathbb{Z}$.

For brevity, we shall omit the superscript $r=1$ throughout. Thus, here and in the sequel we abbreviate

$$
I_{n}=I(h n, i n, j n, k n, l n)=a_{n}-b_{n} \zeta(2),
$$

and we denote again by $M, N$ the integers (2.9).

The transformation formula

$$
I(h, i, j, k, l)=\frac{h ! i !}{(i+j-l) !(l+h-j) !} I(i+j-l, l+h-j, j, k, l)
$$


given in Section 3, corresponding to the permutation $\chi_{2}=\varphi$, yields

$$
\begin{aligned}
I_{n}=a_{n}-b_{n} \zeta(2) & =\frac{(h n) !(i n) !}{((i+j-l) n) !((l+h-j) n) !} I_{n}^{(2)} \\
& =\frac{(h n) !(i n) !}{((i+j-l) n) !((l+h-j) n) !}\left(a_{n}^{(2)}-b_{n}^{(2)} \zeta(2)\right) .
\end{aligned}
$$

It follows that

$$
((i+j-l) n) !((l+h-j) n) ! a_{n}=(h n) !(i n) ! a_{n}^{(2)} .
$$

Let $d_{M n} d_{N n} a_{n}=A_{n}$ and $d_{M^{(2)} n} d_{N^{(2)} n} a_{n}^{(2)}=A_{n}^{(2)}$, whence $A_{n}, A_{n}^{(2)} \in \mathbb{Z}$. Multiplying (4.1) by $d_{M n} d_{N n}$ we get

$$
((i+j-l) n) !((l+h-j) n) ! A_{n}=K(h n) !(i n) ! A_{n}^{(2)},
$$

where

$$
K=\frac{d_{M n} d_{N n}}{d_{M^{(2)} n} d_{N^{(2)} n}}
$$

is an integer since $M^{(2)} \leq M$ and $N^{(2)} \leq N$.

For any prime $p$ and any integer $H>0$ we denote by $v_{p}(H)$ the $p$-adic valuation of $H$, i.e. the exponent of $p$ in the factorization of $H$ into prime powers. Define

$$
\alpha_{p}=v_{p}(((i+j-l) n) !((l+h-j) n) !) \quad \text { and } \quad \beta_{p}=v_{p}((h n) !(i n) !) .
$$

We have

$$
v_{p}(H !)=\sum_{m \geq 1}\left[\frac{H}{p^{m}}\right],
$$

whence $v_{p}(H !)=[H / p]$ if $p>\sqrt{H}$. Since

$$
\begin{aligned}
M & =\max _{1 \leq r \leq 12} M^{(r)} \\
& =\max \{h, i, j, k, l, j+k-h, k+l-i, l+h-j, h+i-k, i+j-l\},
\end{aligned}
$$

for any prime $p$ satisfying

$$
p>\sqrt{M n}
$$

we have

$$
\alpha_{p}=\left[\frac{(i+j-l) n}{p}\right]+\left[\frac{(l+h-j) n}{p}\right]
$$

and

$$
\beta_{p}=\left[\frac{h n}{p}\right]+\left[\frac{i n}{p}\right] .
$$

Let

$$
\omega=\{n / p\},
$$


where $\{x\}=x-[x]$ denotes the fractional part of $x$. For any integer $H$ we have

$$
\frac{H n}{p}=H\left[\frac{n}{p}\right]+H \omega
$$

whence

$$
\left[\frac{H n}{p}\right]=H\left[\frac{n}{p}\right]+[H \omega]
$$

Therefore

$$
\alpha_{p}-\beta_{p}=[(i+j-l) \omega]+[(l+h-j) \omega]-[h \omega]-[i \omega] .
$$

Lemma 4.1. Let $t, u, v, w \in \mathbb{R}$ satisfy $t+u=v+w$. Then

$$
-1 \leq[t]+[u]-[v]-[w] \leq 1 .
$$

Proof. We have

$$
[t]+[u]-[v]-[w]=\{v\}+\{w\}-\{t\}-\{u\},
$$

whence

$$
-2<-\{t\}-\{u\} \leq[t]+[u]-[v]-[w] \leq\{v\}+\{w\}<2 .
$$

By Lemma 4.1 we have $-1 \leq \alpha_{p}-\beta_{p} \leq 1$. Hence, removing from (4.2) the primes $p>\sqrt{M n}$ dividing the factorials on both sides, we obtain

$$
\left(p_{1} \ldots p_{\lambda}\right) P A_{n}=K\left(p_{1}^{\prime} \ldots p_{\lambda^{\prime}}^{\prime}\right) P^{\prime} A_{n}^{(2)},
$$

where $p_{1}, \ldots, p_{\lambda}$ are the distinct primes satisfying (4.3) for which $\alpha_{p}-\beta_{p}=1$, i.e.

$$
[h \omega]+[i \omega]<[(i+j-l) \omega]+[(l+h-j) \omega],
$$

$p_{1}^{\prime}, \ldots, p_{\lambda^{\prime}}^{\prime}$ are the distinct primes satisfying (4.3) for which $\alpha_{p}-\beta_{p}=-1$, i.e.

$$
[(i+j-l) \omega]+[(l+h-j) \omega]<[h \omega]+[i \omega],
$$

and $P, P^{\prime}$ are products of primes $\leq \sqrt{M n}$.

From (4.4) we see that $p_{1}^{\prime}, \ldots, p_{\lambda^{\prime}}^{\prime}$ divide $A_{n}$. Therefore, any prime $p$ satisfying (4.3) and (4.5) divides $A_{n}$.

The above argument applies to each of the transformation formulae in Section 3 corresponding to the permutations $\chi_{2}, \chi_{3}, \chi_{4}, \chi_{5}, \chi_{6}$. Thus we have proved the following 
TheOREM 4.1. Let $\omega=\{n / p\}$. Any prime $p>\sqrt{M n}$ satisfying at least one of the following five inequalities:

$$
\begin{aligned}
{[(i+j-l) \omega]+[(l+h-j) \omega] } & <[h \omega]+[i \omega], \\
{[(j+k-h) \omega]+[(h+i-k) \omega] } & <[i \omega]+[j \omega], \\
{[(k+l-i) \omega]+[(i+j-l) \omega] } & <[j \omega]+[k \omega], \\
{[(l+h-j) \omega]+[(j+k-h) \omega] } & <[k \omega]+[l \omega], \\
{[(h+i-k) \omega]+[(k+l-i) \omega] } & <[l \omega]+[h \omega],
\end{aligned}
$$

divides $A_{n}=d_{M n} d_{N n} a_{n}$.

We now consider the transformation formulae in Section 3 containing three factorials in the numerator and three in the denominator, e.g. the formula corresponding to the permutation $\chi_{7}=\varphi \tau \varphi$. With notation similar to the above, we have

$$
\begin{array}{r}
((k+l-i) n) !((h+i-k) n) !((i+j-l) n) ! A_{n} \\
=L(h n) !(i n) !(j n) ! A_{n}^{(7)},
\end{array}
$$

where $A_{n}^{(7)}=d_{M^{(7)} n} d_{N^{(7)} n} a_{n}^{(7)}$ and $L=d_{M n} d_{N n} /\left(d_{M^{(7)} n} d_{N^{(7)} n}\right)$ are integers.

For any prime $p>\sqrt{M n}$ let

$$
\gamma_{p}=v_{p}(((k+l-i) n) !((h+i-k) n) !((i+j-l) n) !)
$$

and

$$
\delta_{p}=v_{p}((h n) !(i n) !(j n) !)
$$

whence, as before,

$$
\begin{aligned}
\gamma_{p}-\delta_{p} & =[(k+l-i) \omega]+[(h+i-k) \omega]+[(i+j-l) \omega]-[h \omega]-[i \omega]-[j \omega] \\
& =V_{1}+V_{2}
\end{aligned}
$$

where

$$
V_{1}=[(h+i-k) \omega]+[(k+l-i) \omega]-[l \omega]-[h \omega]
$$

and

$$
V_{2}=[l \omega]+[(i+j-l) \omega]-[i \omega]-[j \omega]
$$

satisfy $-1 \leq V_{m} \leq 1(m=1,2)$ by Lemma 4.1 . Therefore $-2 \leq \gamma_{p}-\delta_{p} \leq 2$. Removing from (4.7) the primes $p>\sqrt{M n}$ dividing the factorials, we now have

$$
\left(p_{1} \ldots p_{\lambda}\right)\left(q_{1} \ldots q_{\mu}\right)^{2} P A_{n}=L\left(p_{1}^{\prime} \ldots p_{\lambda^{\prime}}^{\prime}\right)\left(q_{1}^{\prime} \ldots q_{\mu^{\prime}}^{\prime}\right)^{2} P^{\prime} A_{n}^{(7)},
$$

where $p_{1}, \ldots, p_{\lambda} ; q_{1}, \ldots, q_{\mu} ; p_{1}^{\prime}, \ldots, p_{\lambda^{\prime}}^{\prime} ; q_{1}^{\prime}, \ldots, q_{\mu^{\prime}}^{\prime}$ are the distinct primes 
satisfying (4.3) for which $\gamma_{p}-\delta_{p}=1,2,-1,-2$ respectively, and $P, P^{\prime}$ are products of primes $\leq \sqrt{M n}$.

From (4.8) we see that $p_{1}^{\prime}, \ldots, p_{\lambda^{\prime}}^{\prime}$ and $q_{1}^{\prime 2}, \ldots, q_{\mu^{\prime}}^{\prime 2}$ divide $A_{n}$. Therefore, any prime $p>\sqrt{M n}$ for which $\gamma_{p}-\delta_{p}<0$, i.e.

$$
[(k+l-i) \omega]+[(h+i-k) \omega]+[(i+j-l) \omega]<[h \omega]+[i \omega]+[j \omega],
$$

divides $A_{n}$, and any prime $p>\sqrt{M n}$ for which $\gamma_{p}-\delta_{p}=-2$ is such that $p^{2} \mid A_{n}$. Note that the condition $\gamma_{p}-\delta_{p}=-2$ is equivalent to $V_{1}=V_{2}=-1$, i.e. to

$$
\left\{\begin{array}{l}
{[(h+i-k) \omega]+[(k+l-i) \omega]<[l \omega]+[h \omega]} \\
{[l \omega]+[(i+j-l) \omega]<[i \omega]+[j \omega] .}
\end{array}\right.
$$

It is easy to show that any prime $p$ satisfying (4.9) must also satisfy at least one of (4.6), so that (4.9) yields no further primes besides those considered in Theorem 4.1. Hence (4.9), as well as the similar inequalities given by the transformation formulae corresponding to the permutations $\chi_{8}, \chi_{9}, \chi_{10}, \chi_{11}$, can be disregarded. To prove this, let

$$
\begin{aligned}
V_{k} & =[(i+j-l) \omega]+[(l+h-j) \omega]-[h \omega]-[i \omega], \\
V_{l} & =[(j+k-h) \omega]+[(h+i-k) \omega]-[i \omega]-[j \omega], \\
V_{h} & =[(k+l-i) \omega]+[(i+j-l) \omega]-[j \omega]-[k \omega], \\
V_{i} & =[(l+h-j) \omega]+[(j+k-h) \omega]-[k \omega]-[l \omega], \\
V_{j} & =[(h+i-k) \omega]+[(k+l-i) \omega]-[l \omega]-[h \omega],
\end{aligned}
$$

whence

$$
\begin{aligned}
\gamma_{p}-\delta_{p}= & {[(k+l-i) \omega]+[(h+i-k) \omega] } \\
& +[(i+j-l) \omega]-[h \omega]-[i \omega]-[j \omega] \\
= & \frac{1}{2}\left(V_{k}+V_{l}+V_{h}-V_{i}+V_{j}\right) .
\end{aligned}
$$

If (4.9) holds we have $V_{k}+V_{l}+V_{h}+V_{j}<V_{i}$ where, by Lemma 4.1, $V_{k}, V_{l}$, $V_{h}, V_{i}, V_{j}$ would be either 0 or 1 if all the inequalities (4.6) were false. Thus $V_{k}=V_{l}=V_{h}=V_{j}=0, V_{i}=1$, whence $\gamma_{p}-\delta_{p}=\frac{1}{2}\left(V_{k}+V_{l}+V_{h}-V_{i}+V_{j}\right)=$ $-\frac{1}{2}$, a contradiction since $\gamma_{p}-\delta_{p}$ is an integer. Hence (4.9) implies at least one of (4.6).

From (4.10) and the similar inequalities given by the transformation formulae corresponding to $\chi_{8}, \chi_{9}, \chi_{10}, \chi_{11}$ we obtain the following

Theorem 4.2. Let $\omega=\{n / p\}$. Any prime $p>\sqrt{M n}$ satisfying at least one of the following five conditions: 


$$
\begin{aligned}
{[(h+i-k) \omega]+[(k+l-i) \omega] } & <[l \omega]+[h \omega] \quad \text { and } \\
{[l \omega]+[(i+j-l) \omega] } & <[i \omega]+[j \omega], \\
{[(i+j-l) \omega]+[(l+h-j) \omega] } & <[h \omega]+[i \omega] \quad \text { and } \\
{[h \omega]+[(j+k-h) \omega] } & <[j \omega]+[k \omega], \\
{[(j+k-h) \omega]+[(h+i-k) \omega] } & <[i \omega]+[j \omega] \quad \text { and } \\
{[i \omega]+[(k+l-i) \omega] } & <[k \omega]+[l \omega], \\
{[(k+l-i) \omega]+[(i+j-l) \omega] } & <[j \omega]+[k \omega] \quad \text { and } \\
{[j \omega]+[(l+h-j) \omega] } & <[l \omega]+[h \omega], \\
{[(l+h-j) \omega]+[(j+k-h) \omega] } & <[k \omega]+[l \omega] \quad \text { and } \\
{[k \omega]+[(h+i-k) \omega] } & <[h \omega]+[i \omega],
\end{aligned}
$$

is such that $p^{2}$ divides $A_{n}=d_{M n} d_{N n} a_{n}$.

Remark 4.1. If we apply the permutation $\sigma$ to (4.11) we get the conditions:

$$
\begin{aligned}
{[(l+h-j) \omega]+[(j+k-h) \omega] } & <[k \omega]+[l \omega] \quad \text { and } \\
{[l \omega]+[(i+j-l) \omega] } & <[i \omega]+[j \omega], \\
{[(k+l-i) \omega]+[(i+j-l) \omega] } & <[j \omega]+[k \omega] \text { and } \\
{[k \omega]+[(h+i-k) \omega] } & <[h \omega]+[i \omega], \\
{[(j+k-h) \omega]+[(h+i-k) \omega] } & <[i \omega]+[j \omega] \quad \text { and } \\
{[j \omega]+[(l+h-j) \omega] } & <[l \omega]+[h \omega], \\
{[(i+j-l) \omega]+[(l+h-j) \omega] } & <[h \omega]+[i \omega] \quad \text { and } \\
{[i \omega]+[(k+l-i) \omega] } & <[k \omega]+[l \omega], \\
{[(h+i-k) \omega]+[(k+l-i) \omega] } & <[l \omega]+[h \omega] \quad \text { and } \\
{[h \omega]+[(j+k-h) \omega] } & <[j \omega]+[k \omega],
\end{aligned}
$$

which are easily seen to be equivalent to (4.11), since the decomposition $\gamma_{p}-\delta_{p}=V_{1}^{\prime}+V_{2}^{\prime}$, with

$$
V_{1}^{\prime}=[(k+l-i) \omega]+[(i+j-l) \omega]-[j \omega]-[k \omega]
$$

and

$$
V_{2}^{\prime}=[k \omega]+[(h+i-k) \omega]-[h \omega]-[i \omega],
$$

shows that the first of (4.11) is equivalent to the second of (4.12), and similarly for the other conditions. Hence Theorem 4.2, as well as Theorem 4.1, is invariant under the actions of $\boldsymbol{\tau}$ and $\boldsymbol{\sigma}$.

We finally consider the last transformation formula corresponding to the permutation $\chi_{12}$. For any prime $p>\sqrt{M n}$ let

$\varepsilon_{p}=v_{p}(((h+i-k) n) !((k+l-i) n) !((i+j-l) n) !((l+h-j) n) !((j+k-h) n) !)$ 
and

$$
\zeta_{p}=v_{p}((h n) !(i n) !(j n) !(k n) !(l n) !)
$$

whence

$$
\begin{aligned}
\varepsilon_{p}-\zeta_{p}= & {[(h+i-k) \omega]+[(k+l-i) \omega]+[(i+j-l) \omega]+[(l+h-j) \omega] } \\
& +[(j+k-h) \omega]-[h \omega]-[i \omega]-[j \omega]-[k \omega]-[l \omega] \\
= & \frac{1}{2}\left(V_{k}+V_{l}+V_{h}+V_{i}+V_{j}\right) .
\end{aligned}
$$

By Lemma 4.1 we have $-\frac{5}{2} \leq \frac{1}{2}\left(V_{k}+V_{l}+V_{h}+V_{i}+V_{j}\right) \leq \frac{5}{2}$. Since $\varepsilon_{p}-\zeta_{p}$ is an integer, we get $-2 \leq \varepsilon_{p}-\zeta_{p} \leq 2$. If all the inequalities (4.6) are false we have $\varepsilon_{p}-\zeta_{p}=\frac{1}{2}\left(V_{k}+V_{l}+V_{h}+V_{i}+V_{j}\right) \geq 0$. Hence any prime $p$ for which $\varepsilon_{p}-\zeta_{p}<0$ satisfies at least one of (4.6). If $\varepsilon_{p}-\zeta_{p}=-2$ we get $V_{k}+V_{l}+V_{h}+V_{i}+V_{j}=-4$ whence, by Lemma 4.1, four among $V_{k}, V_{l}, V_{h}$, $V_{i}, V_{j}$ are -1 and the remaining one is 0 . If e.g. $V_{k}=V_{l}=V_{h}=V_{j}=-1$, $V_{i}=0$, we obtain $\gamma_{p}-\delta_{p}=\frac{1}{2}\left(V_{k}+V_{l}+V_{h}-V_{i}+V_{j}\right)=-2$, and we have seen that this condition is equivalent to (4.10), i.e. to the first of (4.11). In a similar manner, any other case where $\varepsilon_{p}-\zeta_{p}=-2$ implies one of (4.11). Hence the transformation formula corresponding to $\chi_{12}$ yields no further primes besides those given by Theorems 4.1 and 4.2 .

We now follow Hata's method (see [7], Section 5) to define an integer $D_{n}$ such that $D_{n} \mid d_{M n} d_{N n}$ and $D_{n} a_{n} \in \mathbb{Z}$. To this purpose, however, we employ our Theorems 4.1 and 4.2 in place of Hata's analysis of the $p$-adic valuation of binomial coefficients.

Let $\Omega$ be the set of $\omega \in[0,1)$ satisfying at least one of the inequalities (4.6), and let $\Omega^{\prime}$ be the set of $\omega \in[0,1)$ satisfying at least one of the conditions (4.11). Since each of (4.11) implies one of (4.6), we have $\Omega^{\prime} \subset \Omega$. Clearly $\Omega$ and $\Omega^{\prime}$ are unions of finitely many intervals $\left[\alpha_{q}, \beta_{q}\right)$, where each $\alpha_{q}$ or each $\beta_{q}$ is a rational number whose denominator divides at least one of the integers $h, i, j, k, l, j+k-h, k+l-i, l+h-j, h+i-k, i+j-l$.

Again we assume that the maximal pair $M^{(s)}, N^{(s)}$ considered in Lemma 3.1 is $M^{(1)}=M, N^{(1)}=N$.

Lemma 4.2. If $\omega \in \Omega$ then $\omega \geq 1 / M$. If $\omega \in \Omega^{\prime}$ then $\omega \geq 1 / N$.

Pr o of. Since

$$
\begin{aligned}
M & =\max \{j+k-h, k+l-i, l+h-j, h+i-k, i+j-l\}=\max _{1 \leq r \leq 12} M^{(r)} \\
& =\max \{h, i, j, k, l, j+k-h, k+l-i, l+h-j, h+i-k, i+j-l\},
\end{aligned}
$$

for any $\omega$ satisfying $\omega<1 / M$ we have

$$
\omega<\frac{1}{M} \leq \min \left\{\frac{1}{h}, \frac{1}{i}, \frac{1}{j}, \frac{1}{k}, \frac{1}{l}\right\},
$$


whence

$$
[h \omega]=[i \omega]=[j \omega]=[k \omega]=[l \omega]=0 .
$$

Therefore all the inequalities (4.6) are false, and we get $\omega \notin \Omega$.

Take now any $\omega$ satisfying $\omega<1 / N$. As in the proof of Lemma 3.1 we may assume with no loss of generality that $M=i+j-l$. For otherwise we should replace $h, i, j, k, l$ with $\boldsymbol{\tau}^{m}(h), \boldsymbol{\tau}^{m}(i), \boldsymbol{\tau}^{m}(j), \boldsymbol{\tau}^{m}(k), \boldsymbol{\tau}^{m}(l)$ respectively, where $m$ is such that $M=\tau^{m}(i+j-l)$, and this leaves unchanged the pair $M, N$ as well as the sets $\Omega$ and $\Omega^{\prime}$. By (3.10) we have

$$
\omega<\frac{1}{N} \leq \min \left\{\frac{1}{h}, \frac{1}{i}, \frac{1}{j}, \frac{1}{k}\right\},
$$

whence

$$
[h \omega]=[i \omega]=[j \omega]=[k \omega]=0 .
$$

Therefore

$$
\begin{aligned}
{[l \omega]+[(i+j-l) \omega] } & \geq[i \omega]+[j \omega]=0, \\
{[h \omega]+[(j+k-h) \omega] \geq[j \omega]+[k \omega] } & =0, \\
{[(j+k-h) \omega]+[(h+i-k) \omega] \geq[i \omega]+[j \omega] } & =0, \\
{[(k+l-i) \omega]+[(i+j-l) \omega] \geq[j \omega]+[k \omega] } & =0, \\
{[k \omega]+[(h+i-k) \omega] \geq[h \omega]+[i \omega] } & =0 .
\end{aligned}
$$

Thus all the conditions (4.11) are false, whence $\omega \notin \Omega^{\prime}$.

We now define

$$
\Delta_{n}=\prod_{\substack{p>\sqrt{M n} \\\{n / p\} \in \Omega}} p, \quad \Delta_{n}^{\prime}=\prod_{\substack{p>\sqrt{M n} \\\{n / p\} \in \Omega^{\prime}}} p \quad(n=1,2, \ldots),
$$

where $p$ denotes a prime. From Theorems 4.1 and 4.2 it follows that

$$
\Delta_{n} \Delta_{n}^{\prime} \mid A_{n} .
$$

If $p \mid \Delta_{n}$ we have $\omega=\{n / p\} \in \Omega$, whence $\omega \geq 1 / M$ by Lemma 4.2. Therefore $n / p \geq \omega \geq 1 / M, p \leq M n, p \mid d_{M n}$. Hence

$$
\Delta_{n} \mid d_{M n} .
$$

Similarly, if $p \mid \Delta_{n}^{\prime}$ then $\omega=\{n / p\} \in \Omega^{\prime}$, whence $n / p \geq \omega \geq 1 / N$ again by Lemma 4.2 , and we have $p \leq N n, p \mid d_{N n}$. Hence

$$
\Delta_{n}^{\prime} \mid d_{N n} \text {. }
$$

Thus, if we define

$$
D_{n}=\frac{d_{M n} d_{N n}}{\Delta_{n} \Delta_{n}^{\prime}},
$$


we have $D_{n} \in \mathbb{Z}$ and $D_{n} \mid d_{M n} d_{N n}$ by (4.14) and (4.15), and

$$
D_{n} a_{n}=\frac{A_{n}}{\Delta_{n} \Delta_{n}^{\prime}} \in \mathbb{Z}
$$

by (4.13).

In order to get from this an irrationality measure of $\zeta(2)$, we recall the following well-known lemma (see [8], Lemma 4):

LEMMA 4.3. Let $\alpha \in \mathbb{R}$, and let $\left(r_{n}\right),\left(s_{n}\right)$ be sequences of integers satisfying

and

$$
\lim _{n \rightarrow \infty} \frac{1}{n} \log \left|r_{n}-s_{n} \alpha\right|=-R
$$

$$
\limsup _{n \rightarrow \infty} \frac{1}{n} \log \left|s_{n}\right| \leq S
$$

for some positive numbers $R$ and $S$. Then

$$
\mu(\alpha) \leq \frac{S}{R}+1
$$

Since

$$
D_{n} I_{n}=D_{n} a_{n}-D_{n} b_{n} \zeta(2)
$$

with $D_{n}, b_{n}$ and $D_{n} a_{n}$ integers, we apply Lemma 4.3 with

$$
r_{n}=D_{n} a_{n}, \quad s_{n}=D_{n} b_{n} .
$$

Thus we need

$$
\lim _{n \rightarrow \infty} \frac{1}{n} \log D_{n}, \quad \lim _{n \rightarrow \infty} \frac{1}{n} \log I_{n}, \quad \limsup _{n \rightarrow \infty} \frac{1}{n} \log \left|b_{n}\right| .
$$

Since $d_{M n} d_{N n}=\exp ((M+N) n+o(n))$ by the prime number theorem, we have by (4.16)

$$
\lim _{n \rightarrow \infty} \frac{1}{n} \log D_{n}=M+N-\lim _{n \rightarrow \infty} \frac{1}{n} \log \Delta_{n}-\lim _{n \rightarrow \infty} \frac{1}{n} \log \Delta_{n}^{\prime} .
$$

As is shown by Hata (see [6] and [7]), the last two limits exist and can be easily computed by means of

$$
\psi(x)=\frac{\Gamma^{\prime}(x)}{\Gamma(x)},
$$

the logarithmic derivative of the Euler gamma-function. The result is

$$
\lim _{n \rightarrow \infty} \frac{1}{n} \log \Delta_{n}=\int_{\Omega} d \psi(x), \quad \lim _{n \rightarrow \infty} \frac{1}{n} \log \Delta_{n}^{\prime}=\int_{\Omega^{\prime}} d \psi(x) .
$$

Therefore

$$
\lim _{n \rightarrow \infty} \frac{1}{n} \log D_{n}=M+N-\left(\int_{\Omega} d \psi(x)+\int_{\Omega^{\prime}} d \psi(x)\right) .
$$


5. The irrationality measure of $\zeta(2)$. We now evaluate

$$
\lim _{n \rightarrow \infty} \frac{1}{n} \log I_{n}, \quad \limsup _{n \rightarrow \infty} \frac{1}{n} \log \left|b_{n}\right|,
$$

under the assumption that the integers $h, i, j, k, l, j+k-h, k+l-i$, $l+h-j, h+i-k, i+j-l$ are all strictly positive. Since

$$
I_{n}=I(h n, i n, j n, k n, l n)=\int_{0}^{1} \int_{0}^{1}\left(\frac{x^{h}(1-x)^{i} y^{k}(1-y)^{j}}{(1-x y)^{i+j-l}}\right)^{n} \frac{d x d y}{1-x y},
$$

it is plain that

$$
\lim _{n \rightarrow \infty} \frac{1}{n} \log I_{n}=\max _{0<x, y<1} \log \frac{x^{h}(1-x)^{i} y^{k}(1-y)^{j}}{(1-x y)^{i+j-l}} .
$$

Note that the maximum exists, since by the assumption $h, i, j, k, l>0$ the function

$$
f(x, y)=\frac{x^{h}(1-x)^{i} y^{k}(1-y)^{j}}{(1-x y)^{i+j-l}}
$$

is continuous on the compact square $[0,1] \times[0,1]$, zero on the boundary and positive inside.

From Lemma 2.6 we get, for any $\varrho_{1}, \varrho_{2}>0$ and any $n$,

$$
\begin{aligned}
\frac{1}{n} \log \left|b_{n}\right| & \leq \log \frac{\varrho_{1}^{h}\left(1+\varrho_{1}\right)^{i}\left(1 / \varrho_{1}+\varrho_{2}\right)^{k}\left(1+1 / \varrho_{1}+\varrho_{2}\right)^{j}}{\left(\varrho_{1} \varrho_{2}\right)^{i+j-l}} \\
& =\log \frac{\left(1+\varrho_{1}\right)^{i}\left(1+\varrho_{1} \varrho_{2}\right)^{k}\left(1+\varrho_{1}+\varrho_{1} \varrho_{2}\right)^{j}}{\varrho_{1}^{j+k-h}\left(\varrho_{1} \varrho_{2}\right)^{i+j-l}} .
\end{aligned}
$$

Thus, denoting $\varrho_{1}=u, \varrho_{1} \varrho_{2}=v$, we obtain

$$
\limsup _{n \rightarrow \infty} \frac{1}{n} \log \left|b_{n}\right| \leq \min _{u, v>0} \log \frac{(1+u)^{i}(1+v)^{k}(1+u+v)^{j}}{u^{j+k-h} v^{i+j-l}} .
$$

Again by our assumption the minimum exists, since the function

$$
\frac{(1+u)^{i}(1+v)^{k}(1+u+v)^{j}}{u^{j+k-h} v^{i+j-l}}
$$

is easily seen to be large outside a compact subset of $\{u>0, v>0\}$.

If we make the change of variables $u=-x, v=x y-1$, the function (5.3) becomes

Therefore

$$
(-1)^{h+i+j+k+l} \frac{x^{h}(1-x)^{i} y^{k}(1-y)^{j}}{(1-x y)^{i+j-l}}
$$

$$
\limsup _{n \rightarrow \infty} \frac{1}{n} \log \left|b_{n}\right| \leq \min _{\substack{x, y<0 \\ x y>1}} \log \left|\frac{x^{h}(1-x)^{i} y^{k}(1-y)^{j}}{(1-x y)^{i+j-l}}\right| .
$$


It is easy to see that there are exactly two stationary points of the function (5.2) satisfying $x(1-x) y(1-y) \neq 0$, since the system

$$
\frac{h}{x}-\frac{i}{1-x}+\frac{(i+j-l) y}{1-x y}=\frac{k}{y}-\frac{j}{1-y}+\frac{(i+j-l) x}{1-x y}=0
$$

is equivalent to

$$
\left\{\begin{aligned}
& (l+h-j)(h+i-k) x^{2} \\
& \quad+((l+h-j)(j+k-h)-h(h+i-k)+j(i+j-l)) x \\
& -h(j+k-h)=0 \\
y & =\frac{(h+i-k) x+k-h}{(h+i-k-j) x+j+k-h} .
\end{aligned}\right.
$$

Let $\left(x_{0}, y_{0}\right)$ and $\left(x_{1}, y_{1}\right)$ be the solutions of (5.5), with $0<x_{0}<1$ and $x_{1}<0$. From (5.1) and (5.4) we obtain

$$
\begin{gathered}
\lim _{n \rightarrow \infty} \frac{1}{n} \log I_{n}=\log \frac{x_{0}^{h}\left(1-x_{0}\right)^{i} y_{0}^{k}\left(1-y_{0}\right)^{j}}{\left(1-x_{0} y_{0}\right)^{i+j-l}}, \\
\limsup _{n \rightarrow \infty} \frac{1}{n} \log \left|b_{n}\right| \leq \log \left|\frac{x_{1}^{h}\left(1-x_{1}\right)^{i} y_{1}^{k}\left(1-y_{1}\right)^{j}}{\left(1-x_{1} y_{1}\right)^{i+j-l}}\right| .
\end{gathered}
$$

We can summarize some of the preceding results as follows. Let $h, i, j, k, l$ $>0$ be integers such that

$$
j+k-h, k+l-i, l+h-j, h+i-k, i+j-l>0,
$$

and such that the pair $M=M^{(1)}, N=N^{(1)}$ defined by (2.9) satisfies

$$
M^{(r)} \leq M, \quad N^{(r)} \leq N \quad(r=1, \ldots, 12)
$$

where

$$
\begin{gathered}
M^{(r)}=\max \left\{\chi_{r}(j+k-h), \chi_{r}(k+l-i), \chi_{r}(l+h-j),\right. \\
\left.\chi_{r}(h+i-k), \chi_{r}(i+j-l)\right\}, \\
N^{(r)}=\max \left\{\boldsymbol{\tau}_{r}\left(M^{(r)}\right), \min \left\{\boldsymbol{\tau}_{r}^{2}\left(M^{(r)}\right), \boldsymbol{\tau}_{r}^{3}\left(M^{(r)}\right)\right\}, \boldsymbol{\tau}_{r}^{4}\left(M^{(r)}\right)\right\}
\end{gathered}
$$

is the pair associated with the integral

$$
I^{(r)}=I\left(\chi_{r}(h), \chi_{r}(i), \chi_{r}(j), \chi_{r}(k), \chi_{r}(l)\right),
$$

as is described in Section 3 . Let $\Omega$ be the set of $\omega \in[0,1)$ satisfying at least one of the inequalities (4.6), and let $\Omega^{\prime}$ be the set of $\omega \in[0,1)$ satisfying at least one of the conditions (4.11). Let

$$
f(x, y)=\frac{x^{h}(1-x)^{i} y^{k}(1-y)^{j}}{(1-x y)^{i+j-l}}
$$

let $\left(x_{0}, y_{0}\right)$ and $\left(x_{1}, y_{1}\right)$ be the stationary points of $f(x, y)$ satisfying $x(1-$ $x) y(1-y) \neq 0$, i.e. the solutions of (5.5), with $0<x_{0}, y_{0}<1$ and $x_{1}, y_{1}<0$, and let $\psi(x)=\Gamma^{\prime}(x) / \Gamma(x)$. Denote 


$$
\begin{gathered}
c_{0}=-\log f\left(x_{0}, y_{0}\right), \quad c_{1}=\log \left|f\left(x_{1}, y_{1}\right)\right|, \\
c_{2}=M+N-\left(\int_{\Omega} d \psi(x)+\int_{\Omega^{\prime}} d \psi(x)\right) .
\end{gathered}
$$

Then, by (4.17), (5.6), (5.7) and Lemma 4.3, we get the following

THEOREM 5.1. With the above notation and assumptions we have

$$
\mu(\zeta(2)) \leq \frac{c_{1}+c_{2}}{c_{0}-c_{2}}+1=\frac{c_{0}+c_{1}}{c_{0}-c_{2}},
$$

provided that $c_{0}>c_{2}$.

We now consider some special cases of Theorem 5.1, by making three different choices for $h, i, j, k, l$ which yield good irrationality measures of $\zeta(2)$. We first consider Hata's integral

$$
\int_{0}^{1} \int_{0}^{1}\left(\frac{x^{15}(1-x)^{15} y^{14}(1-y)^{14}}{(1-x y)^{12}}\right)^{n} \frac{d x d y}{1-x y},
$$

through which Hata proved first $\mu(\zeta(2))<6.3489$ (see [7]), and later $\mu(\zeta(2)$ ) $<5.687$ ([7], Addendum) by applying to (5.9) our transformation $\sigma \tau$.

With our notation, the integral $(5.9)$ is $I(15 n, 15 n, 14 n, 14 n, 17 n)$. The pair $M=M^{(1)}, N=N^{(1)}$ associated with

$$
I^{(1)}=I(15,15,14,14,17)
$$

is $M=18, N=16$, and does not satisfy the technical assumption (5.8), since it is easy to check that the maximal pair $M^{(s)}, N^{(s)}$ considered in Lemma 3.1 is now $M^{(s)}=18, N^{(s)}=17$, and is obtained for $s=6$ or $s=11$ (this justifies the exceptional treatment of the interval $\left[\frac{1}{17}, \frac{1}{16}\right)$ in Hata's Addendum [7]). Therefore, in order to apply Theorem 5.1 we must rearrange the pairs $M^{(r)}, N^{(r)}(r=1, \ldots, 12)$ by replacing (5.10) e.g. with

$$
I^{\prime(1)}=I^{(6)}=I(16,16,15,14,14) .
$$

Thus we choose

$$
h=16, \quad i=16, \quad j=15, \quad k=14, \quad l=14,
$$

whence

$j+k-h=13, \quad k+l-i=12, \quad l+h-j=15, \quad h+i-k=18, \quad i+j-l=17$, and the pair $M=18, N=17$ defined by (2.9) satisfies (5.8). The set $\Omega$ is the union of the intervals

$$
\begin{aligned}
& {\left[\frac{1}{16}, \frac{1}{12}\right),\left[\frac{1}{8}, \frac{1}{6}\right),\left[\frac{3}{16}, \frac{4}{17}\right),\left[\frac{1}{4}, \frac{5}{18}\right),\left[\frac{2}{7}, \frac{4}{13}\right),\left[\frac{5}{16}, \frac{1}{3}\right),\left[\frac{5}{14}, \frac{7}{17}\right),\left[\frac{3}{7}, \frac{8}{17}\right),} \\
& {\left[\frac{1}{2}, \frac{7}{13}\right),\left[\frac{9}{16}, \frac{11}{18}\right),\left[\frac{5}{8}, \frac{2}{3}\right),\left[\frac{11}{16}, \frac{12}{17}\right),\left[\frac{5}{7}, \frac{10}{13}\right),\left[\frac{11}{14}, \frac{5}{6}\right),\left[\frac{6}{7}, \frac{8}{9}\right),\left[\frac{13}{14}, \frac{17}{18}\right),}
\end{aligned}
$$

whence

$$
\int_{\Omega} d \psi(x)=9.73032389 \ldots
$$


and the set $\Omega^{\prime}$ is the union of the intervals

$$
\begin{aligned}
& {\left[\frac{1}{14}, \frac{1}{13}\right),\left[\frac{1}{7}, \frac{2}{13}\right),\left[\frac{3}{14}, \frac{2}{9}\right),\left[\frac{3}{8}, \frac{5}{13}\right),\left[\frac{7}{16}, \frac{4}{9}\right),} \\
& {\left[\frac{4}{7}, \frac{7}{12}\right),\left[\frac{9}{14}, \frac{11}{17}\right),\left[\left(\frac{13}{16}, \frac{14}{17}\right),\left[\frac{7}{8}, \frac{15}{17}\right),\left[\frac{15}{16}, \frac{16}{17}\right),\right.}
\end{aligned}
$$

whence

$$
\int_{\Omega^{\prime}} d \psi(x)=1.92426716 \ldots,
$$

so $c_{2}=23.34540893 \ldots$ Also $c_{0}=36.08484700 \ldots, c_{1}=35.34675141 \ldots$ By Theorem 5.1 we obtain

$$
\mu(\zeta(2))<5.607124 \text {. }
$$

As a second example, we make the somewhat simpler choice

$$
h=9, \quad i=8, \quad j=7, \quad k=7, \quad l=9,
$$

whence

$j+k-h=5, \quad k+l-i=8, \quad l+h-j=11, \quad h+i-k=10, \quad i+j-l=6$. The pair $M=11, N=10$ satisfies (5.8). The set $\Omega$ is the union of the intervals

$$
\left[\frac{1}{9}, \frac{1}{5}\right),\left[\frac{2}{9}, \frac{3}{11}\right),\left[\frac{2}{7}, \frac{2}{5}\right),\left[\frac{3}{7}, \frac{1}{2}\right),\left[\frac{5}{9}, \frac{7}{11}\right),\left[\frac{2}{3}, \frac{7}{10}\right),\left[\frac{5}{7}, \frac{9}{11}\right),\left[\frac{6}{7}, \frac{10}{11}\right),
$$

whence

$$
\int_{\Omega} d \psi(x)=7.29482030 \ldots,
$$

and the set $\Omega^{\prime}$ is the union of the intervals

$$
\left[\frac{1}{7}, \frac{1}{6}\right),\left[\frac{4}{9}, \frac{5}{11}\right),\left[\frac{4}{7}, \frac{3}{5}\right),\left[\frac{7}{9}, \frac{4}{5}\right),\left[\frac{8}{9}, \frac{9}{10}\right),
$$

whence

$$
\int_{\Omega^{\prime}} d \psi(x)=1.27367214 \ldots,
$$

so $c_{2}=12.43150754 \ldots$ Here $c_{0}=19.23701154 \ldots, c_{1}=17.82935071 \ldots$

Theorem 5.1 gives

$$
\mu(\zeta(2))<5.446528 \text {. }
$$

Our final example is

$$
h=12, \quad i=12, \quad j=14, \quad k=14, \quad l=13,
$$

whence

$j+k-h=16, \quad k+l-i=15, \quad l+h-j=11, \quad h+i-k=10, \quad i+j-l=13$. The pair $M=16, N=15$ satisfies (5.8). The set $\Omega$ is the union of the intervals

$$
\begin{gathered}
{\left[\frac{1}{14}, \frac{1}{10}\right),\left[\frac{1}{7}, \frac{1}{5}\right),\left[\frac{3}{14}, \frac{3}{11}\right),\left[\frac{2}{7}, \frac{5}{16}\right),\left[\frac{1}{3}, \frac{2}{5}\right),\left[\frac{5}{12}, \frac{7}{15}\right),\left[\frac{1}{2}, \frac{6}{11}\right),} \\
{\left[\frac{4}{7}, \frac{5}{8}\right),\left[\frac{9}{14}, \frac{7}{10}\right),\left[\frac{5}{7}, \frac{11}{15}\right),\left[\frac{3}{4}, \frac{13}{16}\right),\left[\frac{5}{6}, \frac{7}{8}\right),\left[\frac{11}{12}, \frac{15}{16}\right),}
\end{gathered}
$$


whence

$$
\int_{\Omega} d \psi(x)=9.29787398 \ldots,
$$

and the set $\Omega^{\prime}$ is the union of the intervals

$$
\left[\frac{1}{12}, \frac{1}{11}\right),\left[\frac{1}{6}, \frac{2}{11}\right),\left[\frac{5}{14}, \frac{4}{11}\right),\left[\frac{3}{7}, \frac{7}{16}\right),\left[\frac{7}{12}, \frac{3}{5}\right),\left[\frac{11}{14}, \frac{4}{5}\right),\left[\frac{6}{7}, \frac{13}{15}\right),\left[\frac{13}{14}, \frac{14}{15}\right),
$$

whence

$$
\int_{\Omega^{\prime}} d \psi(x)=1.76783442 \ldots
$$

so $c_{2}=19.93429159 \ldots$ Also $c_{0}=31.27178857 \ldots, c_{1}=30.41828189 \ldots$ By

Theorem 5.1 we now have

$$
\mu(\zeta(2))<5.441243
$$

as is stated in the introduction.

\section{References}

[1] F. B e u kers, A note on the irrationality of $\zeta(2)$ and $\zeta(3)$, Bull. London Math. Soc. 11 (1979), 268-272.

[2] A. C. Dixon, On a certain double integral, Proc. London Math. Soc. (2) 2 (1905), $8-15$.

[3] R. Dvornicich and C. Viola, Some remarks on Beukers' integrals, in: Colloq. Math. Soc. János Bolyai 51, Budapest, 1987, 637-657.

[4] A. Erdélyi et al., Higher Transcendental Functions (Bateman Manuscript Project), Vol. 1, McGraw-Hill, New York, 1953.

[5] M. Hata, Legendre type polynomials and irrationality measures, J. Reine Angew. Math. 407 (1990), 99-125.

[6] - Rational approximations to $\pi$ and some other numbers, Acta Arith. 63 (1993), $335-349$.

[7] _- A note on Beukers' integral, J. Austral. Math. Soc. A 58 (1995), 143-153.

[8] G. Rhin and C. Viola, On the irrationality measure of $\zeta(2)$, Ann. Inst. Fourier (Grenoble) 43 (1993), 85-109.

[9] E. A. Rukhadze, A lower bound for the approximation of $\ln 2$ by rational numbers, Vestnik Moskov. Univ. Ser. I Mat. Mekh. 1987 (6), 25-29 (in Russian).

Département de Mathématiques

U.F.R. MIM

Université de Metz

Ile du Saulcy

57045 Metz cedex 1, France

E-mail: rhin@poncelet.univ-metz.fr
Dipartimento di Matematica Università di Pisa Via Buonarroti 2 56127 Pisa, Italy E-mail: viola@dm.unipi.it 